\title{
Optimized Periodic MoM for the Analysis and Design of Dual Polarization Multilayered Reflectarray Antennas Made of Dipoles
}

\author{
Rafael Florencio, Rafael R. Boix, \\ and Giovanni Toso
}

\begin{abstract}
A hybrid version of the Method of Moments (MoM) is applied to the analysis of the scattering of plane waves by periodic multilayered structures containing dipoles at two metallization levels. The MoM matrix entries involving basis functions (BFs) at different metallization levels are computed in the spectral domain as double infinite summations with fast exponential convergence. The MoM matrix entries involving BFs at the same metallization level are computed in the spatial domain as double integrals, which require low-order quadrature rules. The integrands are cross correlations between BFs times multilayered periodic Green's functions (MPGFs). The cross correlations between BFs are obtained in terms of elliptic integrals of first and second kind. Also, the MPGFs are accurately interpolated in 4-D in terms of both the spatial variables and the angles of incidence. The hybrid MoM proposed is used in the design of dual polarization reflectarray antennas under the local periodicity assumption. Thanks to the 4-D interpolation of the MPGFs, which minimizes the total number of MPGFs that have to be computed per reflectarray element, the proposed hybrid MoM is shown to be around 15 times faster than the standard spectral domain MoM in the design of the antennas.
\end{abstract}

Index Terms-Dipole antennas, Green's functions, moment methods, multilayered media, periodic structures, reflectarrays.

\section{INTRODUCTION}

$\mathbf{P}$ RINTED reflectarray antennas uniquely combine the advantages of reflector antennas and printed phased arrays [1]. The main disadvantage of reflectarray antennas comes from its inherent narrow bandwidth. This problem has been alleviated by combining the use of multiresonant elements made of stacked rectangular patches [1], [2] with frequency optimization techniques that enforce the required phase shift in each element in a frequency band rather than
José A. Encinar, a single frequency [3]-[6]. The set of parallel dipoles is an alternative multiresonant reflectarray element showing a flat slope of the phase as a function of dimensions and a phase range that well exceeds $360^{\circ}$ [7]-[12]. The single layer element based on parallel dipoles has been used to design reflectarray antennas with performances that are similar to those attained with the element based on stacked patches for single polarization applications [10]-[12]. For dual polarization applications with frequency reuse, one can arrange two different sets of orthogonal dipoles in two levels of metallization so that the first set controls one polarization, and the orthogonal set controls the other polarization. Since this arrangement provides independent control of the two orthogonal polarizations, the level of cross polarization tends to be very low [13], [14]. In particular, a configuration of this type is proposed in [15], where the independent sets of dipoles for each polarization are shifted half a period. This reflectarray element is prone to frequency optimization techniques, and it leads to an antenna performance comparable to that of the antenna reported in [6], which is based on three stacked patches. But the antenna based on parallel dipoles of [15] has the additional advantage that it has a simpler manufacturing process (three layers needed in [15] versus nine layers needed in [6]) and a lower cross-polarization level [15, Fig. 16].

In the design of a reflectarray antenna made of rectangular patches or dipoles, the dimensions of the patches/dipoles comprising each element have to be adjusted so that the element provides the required phase shift for the generation of a prescribed radiation pattern. In the determination of the dimensions of the patches/dipoles, it is customary to assume that the element is located in a periodic environment [16], [17]. This is called the local periodicity assumption, and its validity is supported because it leads to antenna designs that show good agreement with measurements [5], [6], [18]. The optimized design of a midsize reflectarray antenna made of patches/dipoles in one or two frequency bands may require the numerical analysis of a very large number of different multilayered periodic structures, which indicates that an efficient numerical tool is needed for that purpose. Very recently, Florencio et al. [19] have introduced a hybrid Method of Moments (MoM) formulation for the analysis of multilayered periodic structures containing stacked rectangular patches, the formulation being aimed at reflectarray design. In this paper, basis functions (BFs) with edge singularities are used to model the current density on the rectangular patches. These 
BFs are very convenient for the approximation of the current density in patches with canonical shapes, since they ensure a fast convergence of $\mathrm{MoM}$ with respect to the number of BFs [20]. In the hybrid MoM formulation of [19], the MoM matrix entries involving BFs at different metallization levels are computed in the spectral domain as double infinite summations with fast exponential convergence. However, the MoM matrix entries involving BFs at the same metallization level are not computed in the spectral domain, since they are expressed as slowly convergent double infinite summations in that domain. Instead, these MoM matrix entries are computed in the spatial domain as double finite integrals. The integrands of these double integrals are cross correlations between the BFs (or between their divergences) times multilayered periodic Green's functions (MPGFs). The cross correlations between the BFs are computed in terms of complete elliptic integrals of first and second kind, and the MPGFs are interpolated in 2-D in terms of the two spatial coordinates. The hybrid MoM of [19] has proven to be substantially faster than the traditional spectral MoM when applied to the design of reflectarray antennas made of stacked rectangular patches under the local periodicity assumption.

In this paper, we extend the hybrid MoM numerical tool of [19] to the analysis of multilayered periodic structures containing parallel dipoles at two metallizations levels with a view to applying the implemented tool in reflectarray design. Two main novelties are presented with respect to the approach followed in [19]. First, new cross correlations are derived between the BFs with edge singularities that belong to different dipoles located at the same metallization level. The new cross correlations are needed for the computation of the MoM matrix entries in the spatial domain. They are expressed in terms of complete elliptic functions of first, second, and third kinds [21]. The complete elliptic functions of the third kind are subsequently expressed in terms of normal elliptic functions of first and second kinds, for which efficient computation routines have been developed by Fukushima [22]. Whereas the approach of [19] can only be used for multilayered periodic structures containing one single patch/dipole per metallization level in the unit cell, the approach followed in this paper makes it possible to study multilayered periodic structures containing an arbitrary number of dipoles per metallization level in the unit cell provided the sides of the dipoles are parallel to the boundaries of the unit cell. Second, the MPGFs are not only interpolated in terms of the two spatial coordinates, but also in terms of the two incidence angles of the impinging wave. This 4-D interpolation is carried out in terms of Chebyshev polynomials. Prior to the interpolation, the spectral terms closer to the poles of the MPGFs are subtracted in closed form, because they have a deleterious effect on the interpolation. Whereas the 4-D interpolation does not introduce a significant benefit with respect to the 2-D interpolation of [19] in the analysis of a single periodic structure, the benefits arise in the design of midsize reflectarray antennas, since the 4-D interpolation makes it possible a considerable reduction of the total number of MPGFs that has to be computed per reflectarray element. Thanks to the use of the 4-D interpolation, the results obtained show the developed hybrid MoM turns out to be around

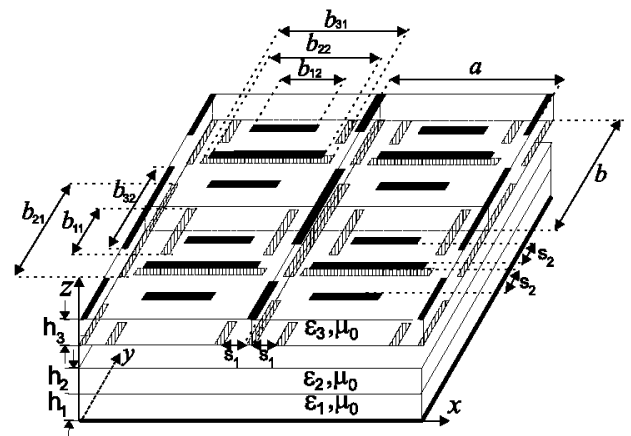

(a)

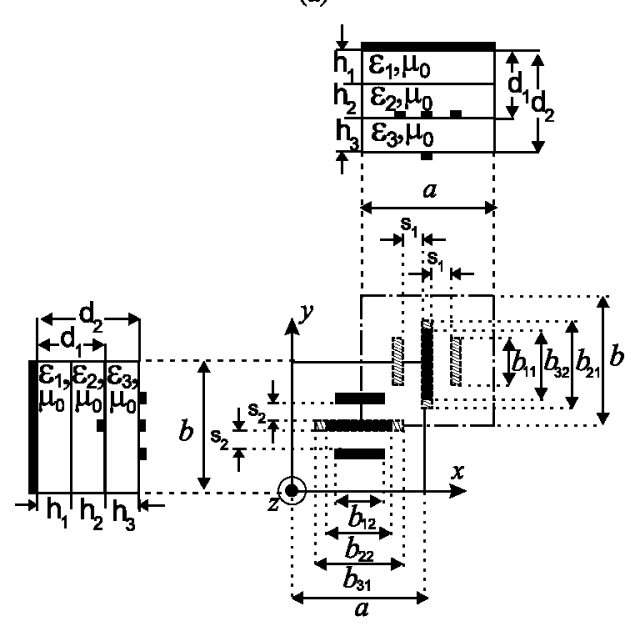

(b)

Fig. 1. Three-layered periodic structure. The unit cell contains two orthogonal sets of four parallel dipoles that are shifted half a period. (a) Exploded view. (b) Side and top views of the expanded unit cell used in the definition of the BFs.

15 times faster than the traditional spectral domain MoM in the design of both focused and contoured beam antennas made of dipoles under the local periodicity assumption.

\section{Numerical PRocedure}

Fig. 1(a) and (b) shows a three-layered periodic structure. The unit cell contains two orthogonal sets of parallel dipoles that are shifted half a period. The dipoles are located at two metallization levels. They are printed at both sides of the upper layer. The intermediate layer is a bonding layer between the upper layer and the lower layer. This lower layer acts as a separator between the upper layer holding the dipoles and the ground plane. The multilayered periodic structure of Fig. 1(a) and (b) was used in [15] to design two dual-polarization reflectarray antennas (one with focused beam and one with contoured beam) under the local periodicity assumption. Other alternative periodic cells containing two orthogonal sets of parallel dipoles at two metallization levels for dual-polarization reflectarray design can be found in [14, Fig. 1], [15, Fig. 2], and [23, Fig. 1]. All these reflectarray cells have in common that they make it possible an independent phase control for each polarization, thus providing low cross polarization. In the following, we will provide a numerical procedure to study the scattering of the periodic structure of Fig. 1(a) and (b) by a plane wave. Without loss of generality, this procedure can be trivially extended to deal 
with other periodic structures whose unit cells contain dipoles at two metallization levels (this is the case of the unit cells shown in [14, Fig. 1], [15, Fig. 2], and [23, Fig. 1]).

\section{A. Hybrid MoM Formulation of the Problem}

Let us assume that the dipoles and ground plane of the periodic structure of Fig. 1 are all PEC of negligible thickness. Also, let $\varepsilon_{i}=\varepsilon_{0} \varepsilon_{r, i}\left(1-j \tan \delta_{i}\right)$ be the complex permittivity of the $i$ th lossy layer of the substrate, and let $h_{i}$ be its thickness $(i=1,2,3)$. For reflectarray design, we need to determine the complex reflection matrix $\mathbf{R}$ of the periodic structure defined in $[15$, eq. 1] when a wave impinges on the structure in an arbitrary incidence direction given by the angular spherical coordinates $\theta_{\text {inc }}$ and $\varphi_{\text {inc }}$. For each incidence direction, the determination of $\mathbf{R}$ requires to obtain the scattered electric field for two different values of the polarization angle $\gamma$ [24, Fig. 1.c]. Assuming a time dependence of the type $e^{j \omega t}$, which will be suppressed throughout, the scattered electric field can be obtained in terms of the current densities $\mathbf{J}_{1}(x, y)$ and $\mathbf{J}_{2}(x, y)$ existing on the two metallized interfaces $z=d_{1}$ and $z=d_{2}$ when the electric field of the wave impinging on the structure is given by (1)-(3) of [24]. These two current densities can be obtained by solving the system of two coupled electric field integral equations (EFIEs) shown as

$$
\begin{gathered}
\hat{\mathbf{z}} \times\left[\mathbf{E}^{\mathrm{ms}}\left(x, y, z=d_{i}\right)+\sum_{j=1}^{2} \sum_{m=-\infty}^{+\infty} \sum_{n=-\infty}^{+\infty} \int_{S_{j}^{m n}}\right. \\
\left.\overline{\mathbf{G}}^{E}\left(x-x^{\prime}, y-y^{\prime}, z=d_{i}, z^{\prime}=d_{j}\right) \cdot \mathbf{J}_{j}\left(x^{\prime}, y^{\prime}\right) d x^{\prime} d y^{\prime}\right]=\mathbf{0} \\
(x, y) \in S_{i}^{00} \quad(i=1,2)
\end{gathered}
$$

where $S_{j}^{m n}(j=1,2 ; m, n=\ldots,-1,0,1, \ldots)$ is the metallized portion of the $z=d_{j}$ plane within the $m n$th unit cell, $\mathbf{E}^{\mathrm{ms}}(x, y, z)$ is the electric field generated in all space by the plane wave impinging on the three-layered substrate in the absence of the dipoles, and $\overline{\mathbf{G}}^{E}$ is the non-periodic dyadic Green's function of the multilayered substrate [25]. Since $\mathbf{J}_{1}(x, y)$ and $\mathbf{J}_{2}(x, y)$ are Floquet-periodic functions [19, eq. 2], in order to solve the system of coupled EFIEs shown in (1), we only need to determine $\mathbf{J}_{1}(x, y)$ and $\mathbf{J}_{2}(x, y)$ within one unit cell. In the particular case of the periodic structure of Fig. 1, we will use the expanded unit cell shown in Fig. 1(b). In that unit cell, we will express $\mathbf{J}_{j}(x, y)(j=1,2)$ in terms of known BFs $\mathbf{J}_{l q}^{j}(x, y)$ as shown in the following:

$$
\mathbf{J}_{j}(x, y)=\sum_{l=1}^{4} \sum_{q=1}^{N_{b}} c_{l q}^{j} \mathbf{J}_{l q}^{j}(x, y) \quad(j=1,2)
$$

where $\mathbf{J}_{l q}^{j}(x, y)\left(j=1,2 ; l=1, \ldots, 4 ; q=1, \ldots, N_{b}\right)$ is the $q$ th $\mathrm{BF}$ of the $l$ th dipole at the $j$ th metallized interface of the expanded unit cell of Fig. 1. When (2) is introduced in (1) and the Galerkin's version of MoM is applied, the unknown coefficients $c_{l q}^{j}$ turn out to be the solution of the following system of linear equations:

$$
\begin{aligned}
\sum_{j=1}^{2} \sum_{l=1}^{4} \sum_{q=1}^{N_{b}} \Omega_{k l, p q}^{i j} c_{l q}^{j}=f_{k p}^{i} \\
\quad\left(i=1,2 ; k=1, \ldots, 4 ; p=1, \ldots, N_{b}\right) .
\end{aligned}
$$

As shown in [19, eq. 7], the MoM matrix entries $\Omega_{k l, p q}^{i j}$ can be obtained in the spectral domain as double infinite summations of the type

$$
\begin{aligned}
\Omega_{k l, p q}^{i j}= & a b \sum_{m=-\infty}^{+\infty} \sum_{n=-\infty}^{+\infty}\left[\left(\widetilde{\mathbf{J}}_{k p}^{i, \mathrm{~d}}\left(k_{x m}, k_{y n}\right)\right)^{*}\right]^{t} \\
& \cdot \widetilde{\overline{\mathbf{G}}}^{E, \mathrm{c}}\left(k_{x}=k_{x m}, k_{y}=k_{y n}, z=d_{i}, z^{\prime}=d_{j}\right) \\
& \widetilde{\mathbf{J}}_{l q}^{j, \mathrm{~d}}\left(k_{x m}, k_{y n}\right) \\
& \quad\left(i, j=1,2 ; k, l=1, \ldots, 4 ; p, q=1, \ldots, N_{b}\right)
\end{aligned}
$$

where $k_{x m}=k_{0} \sin \theta_{\text {inc }} \cos \varphi_{\text {inc }}+2 \pi m / a, \quad k_{y n}=$ $k_{0} \sin \theta_{\text {inc }} \sin \varphi_{\text {inc }}+2 \pi n / b, \widetilde{\mathbf{J}}_{l q}^{j, \mathrm{~d}}\left(k_{x m}, k_{y n}\right)$ stands for the discrete Fourier transform of $\mathbf{J}_{l q}^{j}(x, y)$ as defined in [19, eq. 3], and $\widetilde{\bar{G}}^{E, \mathrm{c}}\left(k_{x}, k_{y}, z=d_{i}, z^{\prime}=d_{j}\right)$ is the continuous 2-D Fourier transform of $\overline{\mathbf{G}}^{E}\left(x, y, z=d_{i}, z^{\prime}=d_{j}\right)$, which can be obtained by means of the recurrent algorithm described in [25]. Also, the constant terms $f_{k p}^{i}$ of the system of equations (3) can be obtained in the spectral domain as [19, eq. 8]

$$
\begin{aligned}
f_{k p}^{i}= & -a b\left[\left(\widetilde{\mathbf{J}}_{k p}^{i, \mathrm{~d}}\left(k_{x 0}, k_{y 0}\right)\right)^{*}\right]^{t} \cdot \mathbf{E}^{\mathrm{ms}}\left(x, y, z=d_{i}\right) \\
& \times e^{-j k_{0}\left(\sin \theta_{\mathrm{inc}} \cos \varphi_{\mathrm{inc}} x+\sin \theta_{\mathrm{inc}} \sin \varphi_{\mathrm{inc}} y\right)} \\
& \left(i=1,2 ; k=1, \ldots, 4 ; p=1, \ldots, N_{b}\right)
\end{aligned}
$$

where the phase term $e^{-j k_{0}\left(\sin \theta_{\text {inc }} \cos \varphi_{\text {inc }} x+\sin \theta_{\text {inc }} \sin \varphi_{\text {inc }} y\right)}$ is included in (5) to absorb the dependence of $\mathbf{E}^{\mathrm{ms}}\left(x, y, z=d_{i}\right)$ on $x$ and $y$.

As commented in [19], the terms involved in the computation of $\Omega_{k l, p q}^{i j}$ show an exponential convergence of the type $e^{-h_{3} \sqrt{k_{x m}^{2}+k_{y n}^{2}}}$ as $k_{x m}^{2}+k_{y n}^{2} \rightarrow \infty$ when $i \neq j$. In the case of the reflectarrays antennas designed in [15], only a few hundred terms are required in (4) to accurately compute $\Omega_{k l, p q}^{i j}$ when $i \neq j$, which means that this computation can be efficiently carried out by means of (4). However, the double infinite summations of (4) converge very slowly when $i=j$. In accordance with [19, eq. 9], this computation should be carried out in the spatial domain by means of the expression

$$
\begin{aligned}
\Omega_{k l, p q}^{i i}=- & j \omega T_{i, k l, p q}^{A}-\frac{1}{j \omega} T_{i, k l, p q}^{\phi} \\
& \quad\left(i=1,2 ; k, l=1, \ldots, 4 ; p, q=1, \ldots, N_{b}\right)
\end{aligned}
$$

where $T_{i, k l, p q}^{A}$ and $T_{i, k l, p q}^{\phi}$ are 2-D integrals, which have to be numerically computed. These integrals are given by

$$
\begin{aligned}
T_{i, k l, p q}^{A}= & \int_{\Gamma_{k l, p q}^{i}} g_{i, k l, p q}^{A}(x, y) \\
& \times G_{x x}^{A, \mathrm{pp}}\left(x, y, z=d_{i}, z^{\prime}=d_{i}\right) d x d y \\
T_{i, k l, p q}^{\phi}= & \int_{\Gamma_{k l, p q}^{i}} g_{i, k l, p q}^{\phi}(x, y) \\
& \times G^{\phi, \mathrm{pp}}\left(x, y, z=d_{i}, z^{\prime}=d_{i}\right) d x d y .
\end{aligned}
$$

The symbol $\Gamma_{k l, p q}^{i}$ of (7) and (8) stands for a rectangular domain whose horizontal (vertical) dimension is the sum of the horizontal (vertical) dimensions of the $k$ th and $l$ th 
dipoles located at $z=d_{i}$. The functions $G_{x x}^{A, \mathrm{pp}}$ and $G^{\phi, \mathrm{pp}}$ are MPGFs with 2-D periodicity, which can be obtained in terms of the spectral Green's functions $\widetilde{G}_{x x}^{A, \mathrm{c}}$ and $\widetilde{G}^{\phi, \mathrm{c}}$ defined in [24, eqs. (13)-(15)] by means of the double infinite summations [19, eqs. 12 and 13]

$$
\begin{aligned}
G_{x x}^{A, \mathrm{pp}}\left(x, y, z=d_{i}, z^{\prime}=d_{i}\right) \\
=\frac{1}{a b} \sum_{m=-\infty}^{+\infty} \sum_{n=-\infty}^{+\infty} \widetilde{G}_{x x}^{A, \mathrm{c}}\left(k_{\rho}=k_{\rho, m n}, z=d_{i}, z^{\prime}=d_{i}\right) \\
\quad \times e^{j\left(k_{x m} x+k_{y n} y\right)} \\
G^{\phi, \mathrm{pp}}\left(x, y, z=d_{i}, z^{\prime}=d_{i}\right) \\
=\frac{1}{a b} \sum_{m=-\infty}^{+\infty} \sum_{n=-\infty}^{+\infty} \widetilde{G}^{\phi, \mathrm{c}}\left(k_{\rho}=k_{\rho, m n}, z=d_{i}, z^{\prime}=d_{i}\right) \\
\quad \times e^{j\left(k_{x m} x+k_{y n} y\right)}
\end{aligned}
$$

where $k_{\rho, m n}=\sqrt{k_{x m}^{2}+k_{y n}^{2}}$. And finally, the functions $g_{i, k l, p q}^{A}$ and $g_{i, k l, p q}^{\phi}$ of (7) and (8) are given by

$$
\begin{aligned}
g_{i, k l, p q}^{A}(x, y)= & \int_{-\infty}^{+\infty} \int_{-\infty}^{+\infty}\left[\left(\mathbf{J}_{k p}^{i}(x+u, y+v)\right)^{*}\right]^{t} \\
& \cdot \mathbf{J}_{l q}^{i}(u, v) d u d v \\
g_{i, k l, p q}^{\phi}(x, y)= & \int_{-\infty}^{+\infty} \int_{-\infty}^{+\infty}\left[\nabla \cdot \mathbf{J}_{k p}^{i}(x+u, y+v)\right]^{*} \\
& \times\left[\nabla \cdot \mathbf{J}_{l q}^{i}(u, v)\right] d u d v .
\end{aligned}
$$

As shown in [19], $g_{i, k l, p q}^{A}(x, y)$ is a 2-D cross correlation between $\mathbf{J}_{k p}^{i}(x, y)$ and $\mathbf{J}_{l q}^{i}(x, y)$, and $g_{i, k l, p q}^{\phi}(x, y)$ is a 2-D cross correlation between $\nabla \cdot \mathbf{J}_{k p}^{i}(x, y)$ and $\nabla \cdot \mathbf{J}_{l q}^{i}(x, y)$. For the particular case of the BFs $\mathbf{J}_{l q}^{i}(x, y)$ used in this paper, the cross correlations of (11) and (12) can be obtained in terms of complete and normal elliptic functions of the first kind and second kind, for which efficient computation routines are available. This makes it possible a fast and accurate computation of both $T_{i, k l, p q}^{A}$ and $T_{i, k l, p q}^{\phi}$ by means of (7) and (8).

\section{B. 4-D Interpolation of the MPGFs}

Let $G_{x x, i}^{A, \mathrm{pp}}\left(k_{x 0}, k_{y 0}, x, y\right)$ and $G_{i}^{\phi, \mathrm{pp}}\left(k_{x 0}, k_{y 0}, x, y\right)$ be $G_{x x}^{A, \mathrm{pP}}\left(x, y, z=d_{i}, z^{\prime}=d_{i}\right)$ and $G^{\phi, \mathrm{pP}}\left(x, y, z=d_{i}, z^{\prime}=d_{i}\right)$ $(i=1,2)$, respectively, where we have explicitly written the dependence of $G_{x x}^{A, \text { pp }}$ and $G^{\phi, \text { pp }}$ on $k_{x 0}$ and $k_{y 0}$. Also, let $\widetilde{G}_{x x, i}^{A, \mathrm{c}}\left(k_{\rho}\right)$ and $\widetilde{G}_{i}^{\phi, \mathrm{c}}\left(k_{\rho}\right)$ be $\widetilde{G}_{x x}^{A, \mathrm{c}}\left(k_{\rho}, z=d_{i}, z^{\prime}=d_{i}\right)$ and $\widetilde{G}^{\phi, \mathrm{c}}\left(k_{\rho}, z=d_{i}, z^{\prime}=d_{i}\right)$, respectively. In [19, eq. (24)], we defined $G_{x x, i}^{A, \mathrm{pp}, \mathrm{reg}}\left(k_{x 0}, k_{y 0}, x, y\right)$ and $G_{i}^{\phi, \mathrm{pp}, \mathrm{reg}}\left(k_{x 0}, k_{y 0}, x, y\right)$, which are regularized versions of $G_{x x, i}^{A, \mathrm{pp}}$ and $G_{i}^{\phi, \mathrm{pp}}$ that were obtained from these MPGFs by extracting their behavior around the sources in closed form. In fact, both the sources singularities and their "complex images" through the closest layers were extracted from the MPGFs [19, eq. 29]. The regularized MPGFs were successfully interpolated in terms of the spatial variables $x$ and $y$ by means of 2-D Chebyshev polynomials [19, eq. 25]. In this paper, we not only intend to interpolate the regularized MPGFs in terms of $x$ and $y$, but also in terms of $k_{x 0}=k_{0} \sin \theta_{\text {inc }} \cos \varphi_{\text {inc }}$ and $k_{y 0}=$ $k_{0} \sin \theta_{\text {inc }} \sin \varphi_{\text {inc }}$, or equivalently, in terms of the incidence angles $\theta_{\text {inc }}$ and $\varphi_{\text {inc }}$. This novel 4-D interpolation is a further step in the efficient design of a reflectarray antenna made of dipoles under the local periodicity assumption by means of the hybrid MoM technique described in Section II-A. Note that a midsize reflectarray antenna is made up of roughly one thousand elements, and that the angles of incidence of the wave impinging on each element from the feed vary from element to element. When the approach of [19, eq. 25] is applied, around one thousand MPGFs interpolated in terms of $x$ and $y$ are required in the design process, one per reflectarray element. However, if the MPGFs are additionally interpolated in terms of $k_{x 0}$ and $k_{y 0}$ (i.e., in terms of $\theta_{\text {inc }}$ and $\varphi_{\text {inc }}$ ), the total number of different MPGFs that has to be computed per reflectarray element is reduced by at least one order of magnitude, which leads to CPU time savings.

Unfortunately, there is a problem with the interpolation of $G_{x x, i}^{A, \text { pp,reg }}$ and $G_{i}^{\phi, \text { pp,reg }}$ in terms of $k_{x 0}$ and $k_{y 0}$, and this problem has to do with the poles of $\widetilde{G}_{x x, i}^{A, \mathrm{c}}\left(k_{\rho}\right)$ and $\widetilde{G}_{i}^{\phi, \mathrm{c}}\left(k_{\rho}\right)$ in the proper Riemann sheet of the complex $k_{p}$ plane [26]. These poles correspond to the propagation constants of TM and TE surface waves above cutoff that can propagate along the threelayered substrate of Fig. 1. In practical cases, the spectral function $\widetilde{G}_{x x, i}^{A, \mathrm{c}}\left(k_{\rho}\right)$ does not usually have any proper pole, since it only has poles related to the propagation constants of TE surface waves, and all these waves are below cutoff for moderately thick substrates and relatively low frequencies [26, Sec. 7.2.2]. However, the spectral function $\widetilde{G}_{i}^{\phi, \mathrm{c}}\left(k_{\rho}\right)$ always has at least one proper pole at $k_{\rho}=k_{\mathrm{TM}_{0}}$ (usually close to $\left.k_{\rho}=k_{0}\right)$, since the proper poles of $\widetilde{G}_{i}^{\phi, \mathrm{c}}\left(k_{\rho}\right)$ come from both TM and TE surface waves, and the $\mathrm{TM}_{0}$ surface wave has zero cutoff frequency. When one analyzes the periodic structure of Fig. 1 under oblique incidence conditions with $\theta_{\text {inc }}>30^{\circ}$, it usually happens that the terms $m=1$ and $n=0, m=-1$ and $n=0, m=0$ and $n=1$, and $m=0$ and $n=-1$ of the double infinite summation (10) lead to values of $k_{\rho}=k_{\rho, m n}$ that are very close to $k_{\rho}=k_{\mathrm{TM}_{0}}$ [27]. This means that the MPGFs $G_{i}^{\phi, p p}$ have a quasi-singular behavior (behavior close to a pole) for certain values of the angles of incidence, and this quasi-singular behavior has a deleterious effect in the interpolation of $G_{i}^{\phi, p p, r e g}\left(k_{x 0}, k_{y 0}, x, y\right)$ in terms of $k_{x 0}$ and $k_{y 0}$. As in [19], this deleterious effect can be avoided if the terms $m=1$ and $n=0, m=-1$ and $n=0$, $m=0$ and $n=1$, and $m=0$ and $n=-1$ of (10) are extracted prior to the interpolation of $G_{i}^{\phi, p p, r e g}\left(k_{x 0}, k_{y 0}, x, y\right)$. For this reason, we are going to define a second version of the regularized Green's function for the scalar potential in the unit cell $\{0 \leq x \leq a ; 0 \leq y \leq b\}, G_{i}^{\phi, \text { pp, reg(2) }}\left(k_{x 0}, k_{y 0}, x, y\right)$, which is given by

$$
\begin{aligned}
& G_{i}^{\phi, \mathrm{pp}, \mathrm{reg}(2)}\left(k_{x 0}, k_{y 0}, x, y\right) \\
& =G_{i}^{\phi, \text { pp,reg }}\left(k_{x 0}, k_{y 0}, x, y\right) \\
& -\frac{1}{a b}\left\{\widetilde{G}_{i}^{\phi, \mathrm{c}}\left(k_{\rho}=k_{\rho, 10}\right) e^{j\left[k_{x 1} x+k_{y 0} y\right]}\right. \\
& +\widetilde{G}_{i}^{\phi, \mathrm{c}}\left(k_{\rho}=k_{\rho,-1,0}\right) \\
& \times e^{j\left[k_{x,-1} x+k_{y 0} y\right]}+\widetilde{G}_{i}^{\phi, \mathrm{c}}\left(k_{\rho}=k_{\rho, 01}\right) e^{j\left[k_{x 0} x+k_{y 1} y\right]} \\
& \left.+\widetilde{G}_{i}^{\phi, \mathrm{c}}\left(k_{\rho}=k_{\rho, 0,-1}\right) e^{j\left[k_{x 0} x+k_{y,-1} y\right]}\right\} \\
& (0 \leq x \leq a ; 0 \leq y \leq b) \text {. }
\end{aligned}
$$


The function $G_{i}^{\phi, \text { pp,reg(2) }}\left(k_{x 0}, k_{y 0}, x, y\right)$ is not any more affected by the pole $k_{\rho}=k_{\mathrm{TM}_{0}}$ of $\widetilde{G}_{i}^{\phi, \mathrm{c}}\left(k_{\rho}\right)$, and presents a smooth behavior for the ranges of angles of incidence $\theta_{\text {inc }}$ and $\varphi_{\text {inc }}$ that are swept in practical reflectarray antennas. Now, let $G_{i}^{\mathrm{pp}, \text { reg }}\left(k_{x 0}, k_{y 0}, x, y\right)$ represent any of the regularized MPGFs $G_{x x, i}^{A, p p, \text { reg }}\left(k_{x 0}, k_{y 0}, x, y\right)$ and $G_{i}^{\phi, p p, r e g(2)}\left(k_{x 0}, k_{y 0}, x, y\right)$. In this paper, we propose to interpolate these regularized MPGFs in terms of 4-D Chebyshev polynomials as shown in the following:

$$
\begin{aligned}
& G_{i}^{\mathrm{pp}, \mathrm{reg}}\left(k_{x 0}, k_{y 0}, x, y\right) \\
& \approx \sum_{r=0}^{N_{c}} \sum_{s=0}^{N_{c}} \sum_{m=0}^{N_{c}} \sum_{n=0}^{N_{c}} h_{r s m n}^{i} \times T_{r}\left(\frac{k_{x 0}}{k_{0} \sin \theta_{\max }}\right) T_{s}\left(\frac{k_{y 0}}{k_{0} \sin \theta_{\max }}\right) \\
& \quad \times T_{m}\left(\frac{2 x}{a}-1\right) \times T_{n}\left(\frac{2 y}{b}-1\right) \\
& \quad\left(-k_{0} \sin \theta_{\max } \leq k_{x 0}, k_{y 0} \leq+k_{0}\right. \\
& \left.\quad \times \sin \theta_{\max } ; 0 \leq x \leq a ; 0 \leq y \leq b\right)
\end{aligned}
$$

where $\theta_{\max }$ is the largest value of $\theta_{\text {inc }}$ involved in the design of a given reflectarray antenna. Although the 4-D interpolation domain of (14) includes values of $k_{x 0}$ and $k_{y 0}$ for which $k_{x 0}^{2}+k_{y 0}^{2} \leq 2 k_{0}^{2} \sin ^{2} \theta_{\max }$, it turns out that $k_{x 0}^{2}+k_{y 0}^{2} \leq$ $k_{0}^{2} \sin ^{2} \theta_{\max }$ in practice. Fortunately, the numerical results obtained indicate that (14) provides interpolated values of $G_{i}^{\mathrm{pp}, \mathrm{reg}}\left(k_{x 0}, k_{y 0}, x, y\right)$ that are sufficiently accurate in the range of values of $k_{x 0}$ and $k_{y 0}$ that are realistic (i.e., those for which $k_{x 0}^{2}+k_{y 0}^{2} \leq k_{0}^{2} \sin ^{2} \theta_{\max }$ ). The coefficients $h_{r s m n}^{i}$ of (14) are given in (15), as shown at the bottom of this page, where $z_{k}=-\cos \left((2 k-1) /\left(2\left(N_{c}+1\right)\right) \pi\right)\left(k=1, \ldots, N_{c}+1\right)$, where

$$
w_{\text {tupq }}^{i}=G_{i}^{\mathrm{pp}, \mathrm{reg}}\left(k_{x 0}=k_{x 0}^{t}, k_{y 0}=k_{y 0}^{u}, x=x_{p}, y=y_{q}\right)
$$

and where $k_{x 0}^{t}=z_{t} k_{0} \sin \theta_{\max }, k_{y 0}^{u}=z_{u} k_{0} \sin \theta_{\max }$, $x_{p}=0.5 a\left(z_{p}+1\right)$ and $y_{q}=0.5 b\left(z_{q}+1\right)(t, u, p, q=$ $\left.1, \ldots, N_{c}+1\right)$.

\section{Basis Functions, Cross Correlations, and Numerical Computation of MoM Matrix Entries in the Spatial Domain}

Fig. 2 shows the $k$ th and $l$ th dipoles located at the interface $z=d_{j}$ of the expanded unit cell drawn in Fig. 1(b). Without loss of generality, we will assume that the $k$ th dipole is oriented along the $x$-direction and the $l$ th dipole along the $y$-direction. The derivations that follow can be easily extended to the case where the two dipoles of Fig. 2 are either two parallel dipoles along the $x$-direction or two parallel dipoles along the $y$-direction.

The BFs chosen to approximate the current density on the dipoles of Fig. 1(b) are products of Chebyshev polynomials

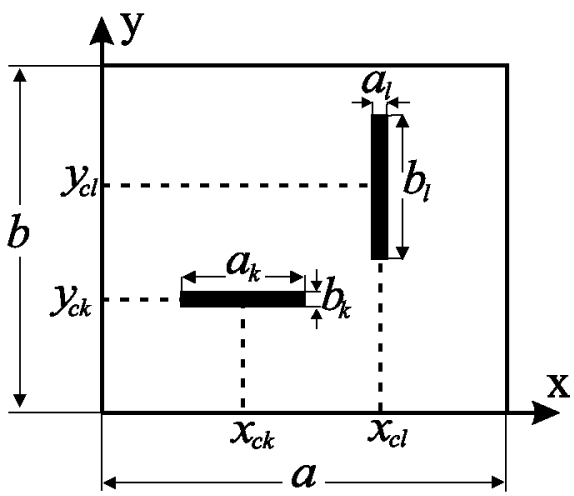

Fig. 2. $k$ th and $l$ th dipoles at the metallized interface $z=d_{j}$ in the expanded unit cell of Fig. 1(b).

weighted by an edge condition, since these BFs ensure a fast convergence of $\mathrm{MoM}$ with respect to the number of $\mathrm{BFs}$ as shown in [19], [20]. In particular, the BFs chosen for the $k$ th dipole of Fig. 2 are

$$
\begin{aligned}
& \mathbf{J}_{k q}^{j}(x, y)-\frac{j k_{0}}{2 q b_{k}} U_{q-1}\left(\frac{2\left(x-x_{c k}\right)}{a_{k}}\right) \\
& \times \sqrt{1-\left(\frac{2\left(x-x_{c k}\right)}{a_{k}}\right)^{2}} \frac{T_{0}\left(\frac{2\left(y-y_{c k}\right)}{b_{k}}\right)}{\sqrt{1-\left(\frac{2\left(y-y_{c k}\right)}{b_{k}}\right)^{2}}} \hat{\mathbf{x}} \\
& \mathbf{J}_{k, q+N_{b 1}}^{j}(x, y) \\
&=-\frac{j k_{0}}{2 q b_{k}} U_{q-1}\left(\frac{2\left(x-x_{c k}\right)}{a_{k}}\right) \\
& \quad \times \sqrt{1-\left(\frac{2\left(x-x_{c k}\right)}{a_{k}}\right)^{2}} \frac{T_{1}\left(\frac{2\left(y-y_{c k}\right)}{b_{k}}\right)}{\sqrt{1-\left(\frac{2\left(y-y_{c k}\right)}{b_{k}}\right)^{2}}} \hat{\mathbf{x}} \\
& \mathbf{J}_{k, N_{b 1}}^{j}+N_{b 2}+1(x, y) \\
&=-\frac{j k_{0}}{4 a_{k}} \frac{T_{1}\left(\frac{2\left(x-x_{c k}\right)}{a_{k}}\right)}{\sqrt{1-\left(\frac{2\left(x-x_{c k}\right)}{a_{k}}\right)^{2}}} \\
& \quad \times U_{1}\left(\frac{2\left(y-y_{c k}\right)}{b_{k}}\right) \sqrt{1-\left(\frac{2\left(y-y_{c k}\right)}{b_{k}}\right)^{2}} \hat{\mathbf{y}}
\end{aligned}
$$

where $T_{p}(\cdot)(p=0,1)$ and $U_{q-1}(\cdot)(q \geq 1)$ are Chebyshev polynomials of first kind and second kind, respectively, and where $N_{b 1}+N_{b 2}+1=N_{b}$. Note that a single BF is used

$$
\begin{aligned}
h_{r s m n}^{i}= & \frac{\sum_{t=1}^{N_{c}+1} \sum_{u=1}^{N_{c}+1} \sum_{p=1}^{N_{c}+1} \sum_{q=1}^{N_{c}+1} w_{t u p q}^{i} T_{r}\left(\mathrm{z}_{t}\right) T_{s}\left(\mathrm{z}_{u}\right) T_{m}\left(\mathrm{z}_{p}\right) T_{n}\left(\mathrm{z}_{q}\right)}{\left[\sum_{t=1}^{N_{c}+1}\left(T_{r}\left(\mathrm{z}_{t}\right)\right)^{2}\right]\left[\sum_{u=1}^{N_{c}+1}\left(T_{s}\left(\mathrm{z}_{u}\right)\right)^{2}\right]} \\
& \times \frac{1}{\left[\sum_{p=1}^{N_{c}+1}\left(T_{m}\left(\mathrm{z}_{p}\right)\right)^{2}\right]\left[\sum_{q=1}^{N_{c}+1}\left(T_{n}\left(\mathrm{z}_{q}\right)\right)^{2}\right]}
\end{aligned}
$$


to model the $y$-component of the current density on the $k$ th dipole, since this component is much less relevant than the $x$-component. The BFs for the $l$ th dipole of Fig. 2 can be easily derived from (17)-(19) if $\left(x-x_{c k}\right)$ and $\left(y-y_{c k}\right)$ are substituted by $\left(y-y_{c l}\right)$ and $\left(x-x_{c l}\right)$, respectively, $b_{k}$ and $a_{k}$ are substituted by $a_{l}$ and $b_{l}$, and $\hat{\mathbf{x}}$ and $\hat{\mathbf{y}}$ are substituted by $\hat{\mathbf{y}}$ and $\hat{\mathbf{x}}$.

In order to obtain the cross correlations of (11) and (12) for the dipoles of Fig. 2, it is essential to express these cross correlations in terms of their Fourier transforms and use convolution theorem. In the case where the two BFs of (11) and (12) belong to the same dipole-e.g., the $k$ th dipole-all the cross correlations can be expressed as linear combinations of functions of the type $t_{m n}\left(x, a_{k}\right) t_{r s}\left(y, b_{k}\right)$, where the integrals $t_{p q}(z, w)$ are defined in [19, eq. 33] and can be computed as shown in [24, eqs. (45)-(53)]. However, in the case where one BF of (11) and (12) belongs to the $k$ th dipole and the other $\mathrm{BF}$ belongs to the $l$ th dipole $-k \neq l-$ the cross correlations cannot be expressed any more in terms of the integrals of [19, eq. 33], and must be written as linear combinations of functions of the type

$$
\begin{aligned}
e_{m n, r s}^{k l}(x, y)= & I_{m n}\left(2\left(x_{c k}-x_{c l}-x\right), a_{k}, a_{l}\right) \\
& \times I_{r s}\left(2\left(y_{c k}-y_{c l}-y\right), b_{k}, b_{l}\right) \\
& (k, l=1,2 ; k \neq l ; m, n, r, s=1,2,3, \ldots) .
\end{aligned}
$$

where the functions $I_{p q}(z, u, v)$ are expressed in terms of the new integrals

$$
\begin{array}{r}
I_{p q}(z, u, v)=j^{p-q} \int_{-\infty}^{+\infty} J_{p-1}(u w) J_{q-1}(v w) e^{j z w} d w \\
(p, q=1,2,3, \ldots) .
\end{array}
$$

In $(21), J_{p-1}(\cdot)\left(J_{q-1}(\cdot)\right)$ are Bessel functions of first kind and order $p-1(q-1)$. The determination of $e_{m n, r s}^{k l}(x, y)$ $(k \neq l)$ requires the determination of $I_{p q}(z, u, v)$ for $u>0$ and $v>0$. Since $I_{p q}(z, u, v)$ turns out to be zero when $z \leq-(u+v)$ and $z \geq+(u+v)$, this determination is limited to the interval $-(u+v)<z<+(u+v)$ for practical purposes. In order to obtain $I_{p q}(z, u, v)$ when $u>0, v>0$, and $|z|<u+v$, we need to define four parameters $a, b, c$, and $d$ in terms of $z, u$, and $v$ as shown in Appendix A. It should be pointed out that these four parameters only make sense when $u \neq v$, since the computation of $I_{p q}(z, u, v)$ when $u=v$ reduces to the case treated in [24, eqs. (45)-(53)]. Once the four parameters $a, b, c$, and $d$ have been defined in terms of $z, u$, and $v$ (see Appendix A), both convolution theorem and [28, eq. (7.355)] can be used to transform the integrals of (21) into

$$
I_{p q}(\mathrm{z}, u, v)=\frac{2}{\pi v} \int_{c}^{b} \frac{T_{p-1}\left(\frac{v s^{\prime}-z}{u}\right) T_{q-1}\left(s^{\prime}\right) d s^{\prime}}{\sqrt{\left(a-s^{\prime}\right)\left(b-s^{\prime}\right)\left(s^{\prime}-c\right)\left(s^{\prime}-d\right)}}
$$

where $T_{p-1}$ and $T_{q-1}$ are again Chebyshev polynomials of the first kind. Note that the numerator of the integrand in (22) is a polynomial of degree $p+q-2$ that can be written as

$$
T_{p-1}\left(\frac{v s^{\prime}-z}{u}\right) T_{q-1}\left(s^{\prime}\right)=\sum_{n=0}^{p+q-2} d_{n}(z, u, v)\left(s^{\prime}\right)^{n} .
$$

When (23) is introduced in (22), the following expression for $I_{p q}(z, u, v)$ is obtained:

$$
I_{p q}(z, u, v)=\frac{2}{\pi v} \sum_{n=0}^{p+q-2} d_{n}(z, u, v) J_{n}(a, b, c, d)
$$

where

$$
\begin{aligned}
& J_{n}(a, b, c, d)=\int_{c}^{b} \frac{\left(s^{\prime}\right)^{n} d s^{\prime}}{\sqrt{\left(a-s^{\prime}\right)\left(b-s^{\prime}\right)\left(s^{\prime}-c\right)\left(s^{\prime}-d\right)}} \\
& (n \geq 0) \text {. }
\end{aligned}
$$

The integrals $J_{n}(a, b, c, d)$ can be obtained as shown in Appendix B. In fact, this computation only requires the determination of complete and normal elliptic integrals of first kind and second kind. These elliptic integrals can all be obtained by using the efficient methods recently developed by Fukushima [22] (see [22] and references therein). In fact, numerical simulations have shown that the routines based on Fukushima's algorithms for the numerical computation of elliptic integrals are typically three times faster than those supplied in [29]. Apart from the use of Fukushima's algorithms, the computational burden involved in the determination of the integrals $I_{p q}(z, u, v)$ can be alleviated if one makes use of the recurrence relations shown in Appendix C.

Since the integrals $I_{p q}(z, u, v)$ of (21) are only different from zero in the interval $-(u+v)<z<+(u+v)$, the functions $e_{m n, r s}^{k l}(x, y)$ of (20)-and therefore, the cross correlations $g_{i, k l, p q}^{A}(x, y)$ and $g_{i, k l, p q}^{\phi}(x, y)$ of (11) and (12)will accordingly be different from zero in the rectangular domain of the $x y$ plane

$$
\begin{aligned}
& \left\{x_{1}^{k l}=x_{3}^{k l}-\frac{a_{k}+a_{l}}{2}<x<x_{5}^{k l}=x_{3}^{k l}+\frac{a_{k}+a_{l}}{2}\right\} \\
& \quad \times\left\{y_{1}^{k l}=y_{3}^{k l}-\frac{b_{k}+b_{l}}{2}<y<y_{5}^{k l}=y_{3}^{k l}+\frac{b_{k}+b_{l}}{2}\right\}
\end{aligned}
$$

where $x_{3}^{k l}=x_{c k}-x_{c l}$ and $y_{3}^{k l}=y_{c k}-y_{c l}$. The rectangular domain of (26) turns out to be the integration domain $\Gamma_{k l, p q}^{i}$ of (7) and (8).

When the cross correlations $g_{i, k l, p q}^{A}(x, y)$ and $g_{i, k l, p q}^{\phi}(x, y)$ are cross correlations between BFs belonging to the same dipole-e.g., the $k$ th dipole - the integration domain $\Gamma_{k k, p q}^{i}$ includes the point $(x=0, y=0)$ as shown in [19, eq. 32] [see also (26) in the particular case where $k=l$ ]. Since the integrand of (7) and (8) is singular at $(x=0, y=0)$, this singular behavior has to be extracted $[19$, eqs. 37 to 39] and carefully integrated as discussed in [19, App. B]. Also, the integrands of (7) and (8) present logarithmic singularities in the integration domain $\Gamma_{k k, p q}^{i}$ that can be adequately handled by using Ma-Rokhlin-Wandzura (MRW) quadrature rules [30]. In the case where the cross correlations $g_{i, k l, p q}^{A}(x, y)$ and $g_{i, k l, p q}^{\phi}(x, y)$ are cross correlations between $\mathrm{BFs}$ belonging to different dipoles $(k \neq l)$, the integration domain $\Gamma_{k l, p q}^{i}$ does not include 


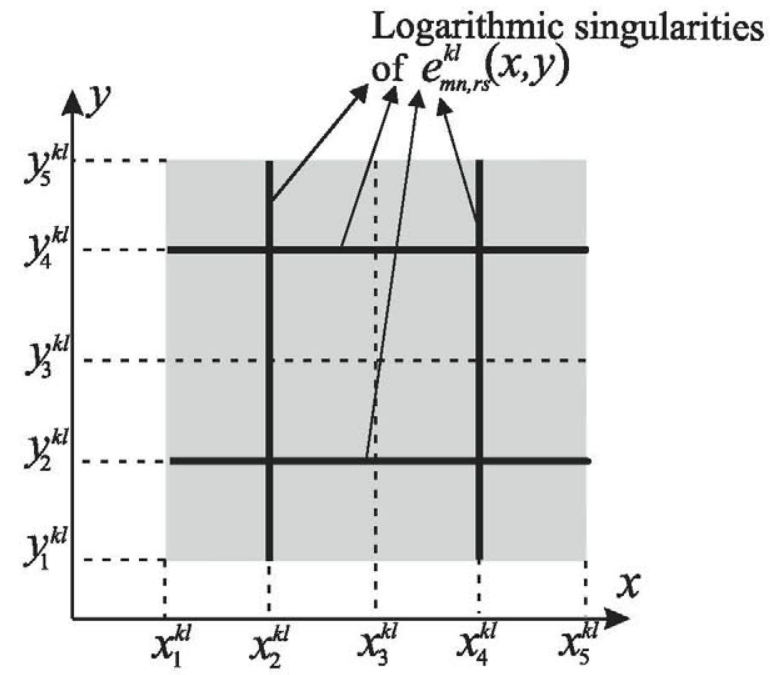

Fig. 3. Integration domain $\Gamma_{k l, p q}^{i} \equiv\left\{x_{1}^{k l}<x<x_{5}^{k l}\right\} \times\left\{y_{1}^{k l}<y<y_{5}^{k l}\right\}$ of (7) and (8) when $k \neq l$. The location of the logarithmic singularities within the integration domain is clearly indicated.

anymore the point $(x=0, y=0)$, and therefore, there is no need to extract the singular behavior at $(x=0, y=0)$ when carrying out the numerical integration of (7) and (8). However, the cross correlations $g_{i, k l, p q}^{A}(x, y)$ and $g_{i, k l, p q}^{\phi}(x, y)$ present logarithmic singularities in the integration domain $\Gamma_{k l, p q}^{i}$, even when $k \neq l$. These singularities are introduced by the complete elliptic integrals $\boldsymbol{K}(k)$ and $\Pi\left(\alpha^{2}, k\right)$ of (39)-(41), which present logarithmic singularities when $k=1$. According to (42), this happens when $a=b$ or $c=d$ in (42). This means that the integrals $J_{n}(a, b, c, d)$ of (25) will be singular when $a=b$ or $c=d$. And according to the definitions of $a, b, c$, and $d$ provided in Appendix A, the logarithmic singularities of $J_{n}(a, b, c, d)$ will appear in the integrals $I_{p q}(z, u, v)$ when $z=u-v$ and $z=v-u$. Bearing in mind (20), these logarithmic singularities will also be present in the functions $e_{m n, r s}^{k l}(x, y)$-and therefore, in the cross correlations $g_{i, k l, p q}^{A}(x, y)$ and $g_{i, k l, p q}^{\phi}(x, y)$-when

$$
\begin{aligned}
& x=x_{2}^{k l}=x_{3}^{k l}-\left|\frac{a_{k}-a_{l}}{2}\right| \\
& x=x_{4}^{k l}=x_{3}^{k l}+\left|\frac{a_{k}-a_{l}}{2}\right| \\
& y=y_{2}^{k l}=y_{3}^{k l}-\left|\frac{b_{k}-b_{l}}{2}\right| \\
& y=y_{4}^{k l}=y_{3}^{k l}+\left|\frac{b_{k}-b_{l}}{2}\right| .
\end{aligned}
$$

It turns out that $x_{1}^{k l}<x_{2}^{k l}<x_{4}^{k l}<x_{5}^{k l}$ and $y_{1}^{k l}<y_{2}^{k l}<y_{4}^{k l}<y_{5}^{k l}$, which indicates that the logarithmic singularities of $e_{m n, r s}^{k l}(x, y)$ at $x=x_{2}^{k l}, x=x_{4}^{k l}, y=y_{2}^{k l}$, and $y=y_{4}^{k l}$ will appear in the integration domain $\Gamma_{k l, p q}^{i}$ of (7) and (8). Fig. 3 shows a clear picture of this integration domain and of the location of the logarithmic singularities.

In order to carry out the numerical computation of the integrals (7) and (8) when $k \neq l$, the rectangular integration domain $\Gamma_{k l, p q}^{i} \equiv\left\{x_{1}^{k l}<x<x_{5}^{k l}\right\} \times\left\{y_{1}^{k l}<y<y_{5}^{k l}\right\}$ of each

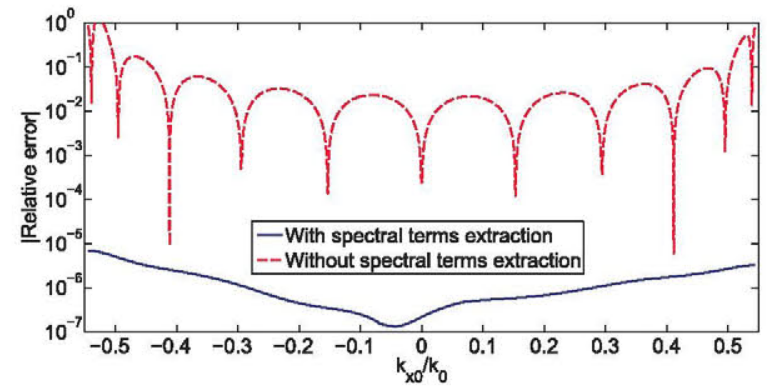

Fig. 4. Magnitude of the relative errors between the spatial domain MPGF for the scalar potential $G^{\phi, p p}$ and the interpolation obtained from (14) (as a function of $k_{x 0}$ for fixed values of $k_{y 0}, x$, and $y$ ). Results are presented for the case where the spectral domain terms are not extracted prior to the interpolation as in [19, eq. (24)] (dashed line), and for the case where the spectral domain terms are extracted prior to the interpolation as in (13) (solid line). Parameters: $z=z^{\prime}=d_{1}=h_{1}+h_{2}=2.439 \mathrm{~mm}, f=14$ $\mathrm{GHz}, a=b=12 \mathrm{~mm}, x=0.4 a, y=0.4 b, \theta_{\max }=33^{\circ}, \varphi_{\text {inc }}=0^{\circ}$, and $N_{c}=10$.

integral is divided into the 16 rectangular subdomains $\left\{x_{i}^{k l}<\right.$ $\left.x<x_{i+1}^{k l}\right\} \times\left\{y_{j}^{k l}<y<y_{j+1}^{k l}\right\}(i, j=1,2,3,4)$, each of which has two sides with logarithmic singularities (see Fig. 3). And each integral is expressed as the summation of 16 subintegrals, each of which is defined over one of the 16 integration subdomains. After that, a convenient change of variables is applied to each subintegral so that it is converted into a new subintegral in the square domain $\{0<u<1\} \times\{0<v<1\}$ with logarithmic singularities at $u=0$ and $v=0$. Finally, all these subintegrals are collected together into one single integral in the square domain $\{0<u<1\} \times\{0<v<1\}$ as in [19, eq. (40)], and this single integral with logarithmic singularities at $u=0$ and $v=0$ is efficiently computed by iterated application of MRW quadrature rules [30] as in [19].

\section{NUMERICAL RESUlTS AND VALIDATIONS}

The three-layered substrate employed to obtain the results presented in this section is that used for the design of the reflectarray antennas of [15]. For this substrate, $h_{1}=2.363$ $\mathrm{mm}, h_{2}=0.076 \mathrm{~mm}, h_{3}=1.5 \mathrm{~mm}, \varepsilon_{r, 1}=2.55, \varepsilon_{r, 2}=2.32$, $\varepsilon_{r, 3}=2.17, \tan \delta_{1}=0.0009, \tan \delta_{2}=0.0013$, and $\tan \delta_{3}=$ 0.0009 . The thin intermediate layer is a bonding layer used to glue the upper and lower layers. This thin layer is conveniently handled in MPGF interpolations as explained in [19, Sec. III].

Fig. 4 shows results for the errors arising in the interpolation of the MPFG for the scalar potential $G^{\phi, p p}$ as the variable $k_{x 0}$ of (14) changes and the other three variables $-k_{y 0}, x$ and $y$-remain fixed (similar results have been obtained when $k_{y 0}$ changes, while $k_{x 0}, x$, and $y$ remain fixed). The dashed line corresponds to the case where the regularized Green's function $G_{1}^{\phi, \text { pp,reg }}\left(k_{x 0}, k_{y 0}, x, y\right)$ of [19, eq. (24)] is used in the interpolation of (14), and the solid line corresponds to the case where the regularized Green's function $G_{1}^{\phi, p p, \operatorname{reg}(2)}\left(k_{x 0}, k_{y 0}, x, y\right)$ of (13) is used in (14). Note that when the spectral terms $m=1$ and $n=0, m=-1$ and $n=0, m=0$ and $n=1$, and $m=0$ and $n=-1$ of (10) are not extracted prior to interpolation, the errors in $G^{\phi, \mathrm{pp}}$ become larger than $10 \%$ in spite of the fact that a large number of Chebyshev polynomials is used in the interpolation $-N_{c}=10$. However, when the spectral terms are extracted, the interpolation errors 


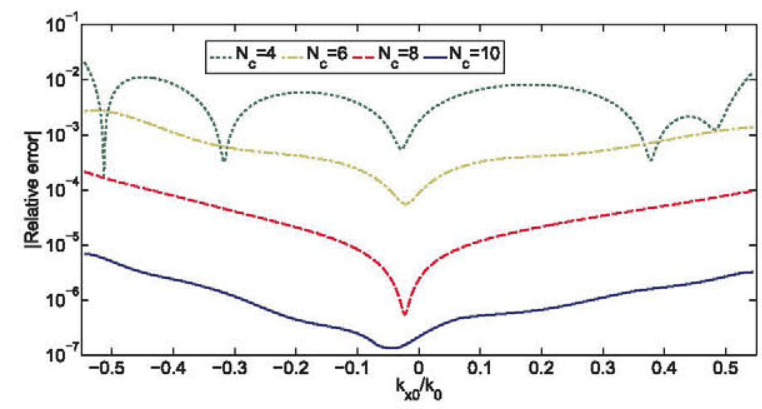

Fig. 5. Magnitude of the relative errors between the spatial domain MPGF for the scalar potential $G^{\phi, \mathrm{pp}}$ and the interpolation obtained from (13) and (14) (as a function of $k_{x 0}$ for fixed values of $k_{y 0}, x$, and $y$ ). Results are presented for different values of the number of Chebyshev polynomials $N_{C}$ used for each of the four interpolation variables. Parameters: $z=z^{\prime}=d_{1}=h_{1}+h_{2}=$ $2.439 \mathrm{~mm}, f=14 \mathrm{GHz}, a=b=12 \mathrm{~mm}, x=0.4 a, y=0.4 b, \theta_{\max }=33^{\circ}$, $\varphi_{\text {inc }}=0^{\circ}$, and $N_{c}=10$.

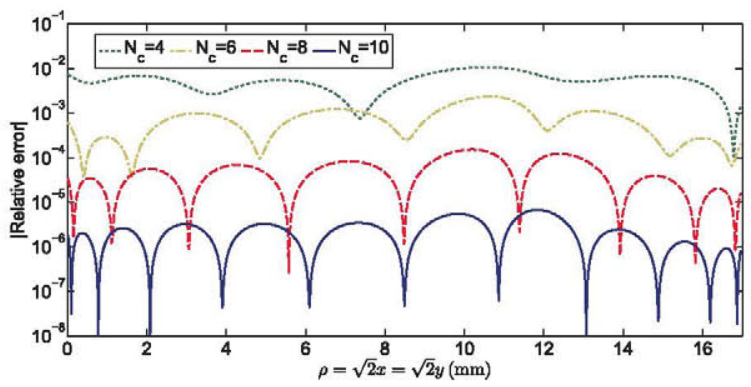

Fig. 6. Magnitude of the relative errors between the spatial domain MPGF for the scalar potential $G^{\phi, p p}$ and the interpolation obtained from (13) and (14). The MPFG values are sampled along the diagonal of the unit cell (for fixed values of $k_{x 0}$ and $k_{y 0}$ ). Results are presented for different values of the number of Chebyshev polynomials $N_{C}$ used for each of the four interpolation variables. Parameters: $z=z^{\prime}=d_{1}=h_{1}+h_{2}=2.439 \mathrm{~mm}, f=14 \mathrm{GHz}, a=b=$ $12 \mathrm{~mm}, \theta_{\text {inc }}=30^{\circ}, \varphi_{\text {inc }}=0^{\circ}$, and $N_{c}=10$.

become smaller than $0.001 \%$ when $N_{c}=10$. As commented in Section II, this behavior is caused by the fact that the spectral terms $m=1$ and $n=0, m=-1$ and $n=0, m=0$ and $n=1$, and $m=0, n=-1$ of (10) introduce quasisingularities in $G^{\phi, \mathrm{pp}}$, since the values of $k_{\rho, 10}, k_{\rho, 01}, k_{\rho,-1,0}$, and $k_{\rho, 0,-1}$ become close to the spectral domain pole $k_{\mathrm{TM}_{0}}$ as the angle of incidence $\theta_{\text {inc }}$ approaches $\theta_{\max }$ (although in practical reflectarrays, you try to avoid this pole to prevent the excitation of surface waves in the substrate, the discretized spectral variable $k_{\rho, m n}$ unavoidably approaches the pole as the angle of incidence $\theta_{\text {inc }}$ increases [31]). And these quasisingularities have a harmful effect on the interpolation with polynomials. As soon as the quasi-singularities are removed from the function to be interpolated, the interpolation works correctly as shown in Fig. 4. In the case of the MPGF for the vector potential, $G_{x x}^{A, \mathrm{pp}}$, we have obtained interpolation errors below $0.001 \%$ when $N_{c}=10$ (i.e., as in the solid line of Fig. 4) without extracting the spectral terms $m=1$ and $n=0, m=-1$ and $n=0, m=0$ and $n=1$, and $m=0$ and $n=-1$ of (10). The explanation for this is that the pole $k_{\mathrm{TM}_{0}}$ is not present in $\widetilde{G}_{x x, 1}^{A, \mathrm{c}}\left(k_{\rho}\right)$, which is the spectral domain version of $G_{x x}^{A, \mathrm{pp}}$.

Figs. 5 and 6 show the convergence in the interpolation errors of $G^{\phi, p p}$ as the number of Chebyshev polynomials $N_{c}$ increases. Equations (13) and (14) are used in the interpolation

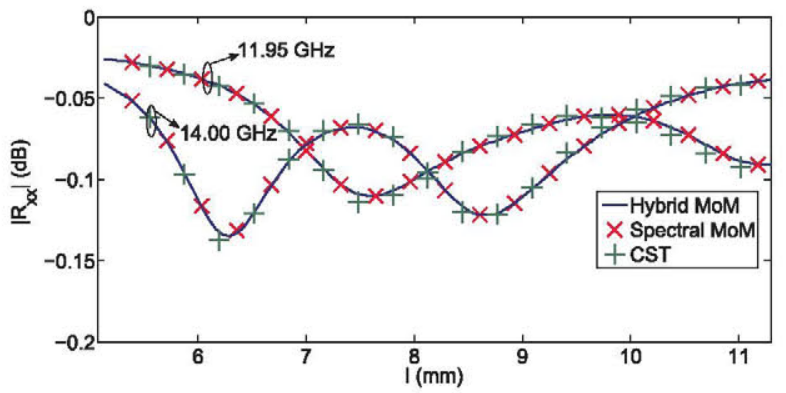

(a)

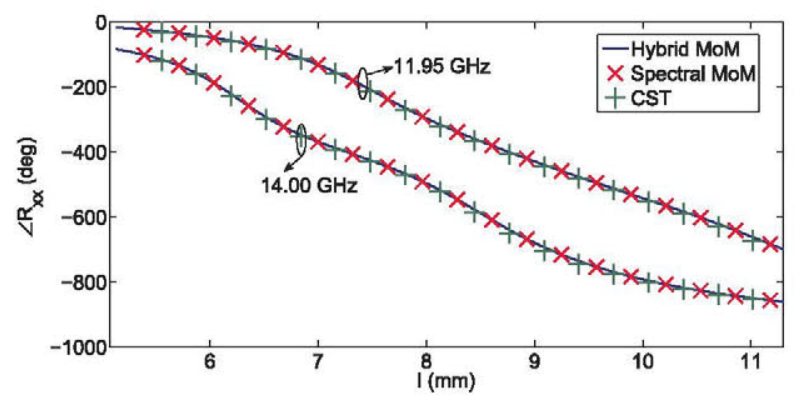

(b)

Fig. 7. (a) Magnitude and (b) phase of $R_{x x}$ for the reflectarray element made of two orthogonal sets of four dipoles that has been used in [15]. Our results obtained with both the spectral domain $\mathrm{MoM}(\times)$ and the hybrid MoM of Section II (solid lines) are compared with those obtained with CST (+). Parameters: $a=b=12 \mathrm{~mm}, \theta_{\text {inc }}=16.9^{\circ}, \varphi_{\text {inc }}=0^{\circ}$, dipole width $w=0.5 \mathrm{~mm}, s_{1}=s_{2}=3.5 \mathrm{~mm}, b_{11}=0.58 b_{21}, b_{32}=0.95 b_{21}$, $b_{22}=0.93 b_{31}, b_{12}=0.63 b_{31}$, and $b_{31}=b_{21}=l$.

of $G^{\phi, p p}$ to avoid the harmful effect of the quasi-singularities of (10). In Fig. 5, we study the convergence of the interpolation errors as a function of $k_{x 0}$ for fixed values of $k_{y 0}, x$, and $y$ (in particular, in this case, $k_{x 0} / k_{0}=\sin \theta_{\text {inc }}$ and $k_{y 0}=0$, since $\varphi_{\text {inc }}$ has been assumed to be $0^{\circ}$ in the plotted curves). And in Fig. 6 , we study this convergence as a function of $x=y$ for fixed values of $k_{x 0}$ and $k_{y 0}$. A similar level of interpolation errors can be observed in both Figs. 5 and 6. Thus, it can be stated that three significant figures are roughly achieved in the interpolated values of $G^{\phi, \mathrm{pp}}$ when $N_{c}=6$, four significant figures are roughly achieved when $N_{c}=8$, and five significant figures are achieved when $N_{c}=10$. The convergence in the interpolation errors of $G^{\phi, p p}$ with respect to $N_{c}$ for the four interpolation variables involved in (14) demonstrates the regularized Green's function $G_{1}^{\phi, p p, r e g(2)}\left(k_{x 0}, k_{y 0}, x, y\right)$ of (13) is a smooth function of all these variables. In the rest of this paper, we will use $N_{c}=7$ for the interpolations, which suffices to ensure between three and four significant figures in the interpolated values of the MPGFs.

In order to validate our MoM codes for the periodic structure with two orthogonal sets of four parallel dipoles of Fig. 1, in Fig. 7(a) and (b), the magnitude and phase of the $R_{x x}$ element of the reflection matrix (see [15, eq. 1]) are plotted for the particular structure analyzed in [15, Fig. 7]. The phases of $R_{x x}$ and $R_{y y}$ are the crucial parameters in the design of a dual-polarization reflectarray antenna [15], and in this case, the phase range achieved for $R_{x x}$ is close to $700^{\circ}$. The magnitude of $R_{x x}$ is used to estimate the dielectric losses 


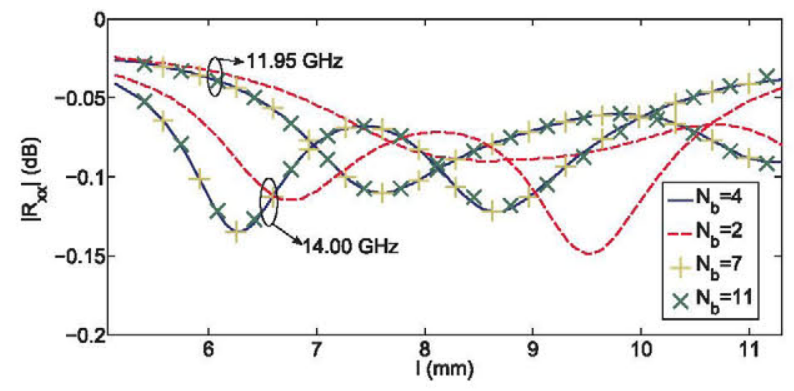

(a)

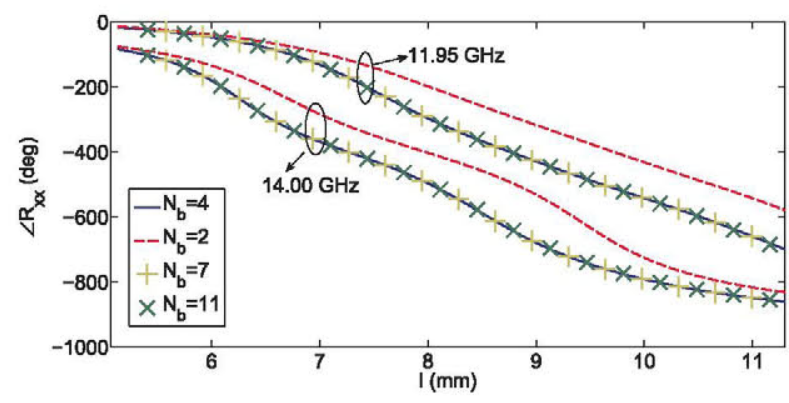

(b)

Fig. 8. (a) Magnitude and (b) phase of $R_{x x}$ for the reflectarray element made of two orthogonal sets of four dipoles that has been used in [15]. Convergence with respect to the number of BFs $N_{b}$ employed in the approximation of the current density on the dipoles. Dashed line is used for $N_{b}=2\left(N_{b 1}=1\right.$ and $\left.N_{b 2}=0\right)$, solid line for $N_{b}=4\left(N_{b 1}=3\right.$ and $\left.N_{b 2}=0\right)$, the symbol + is used for $N_{b}=7\left(N_{b 1}=N_{b 2}=3\right)$, and the symbol $\times$ for $N_{b}=11$ $\left(N_{b 1}=N_{b 2}=5\right)$. Parameters: $a=b=12 \mathrm{~mm}, \theta_{\text {inc }}=16.9^{\circ}, \varphi_{\text {inc }}=0^{\circ}$, dipole width $w=0.5 \mathrm{~mm}, s_{1}=s_{2}=3.5 \mathrm{~mm}, b_{11}=0.58 b_{21}, b_{32}=0.95 b_{21}$, $b_{22}=0.93 b_{31}, b_{12}=0.63 b_{31}$, and $b_{31}=b_{21}=l$.

in the designed antenna. In Fig. 7(a) and (b), the results obtained with the standard spectral domain MoM [i.e., (4) is used for the determination of MoM matrix entries when $i, j=1,2]$ are compared with results obtained with the hybrid spatial/spectral MoM described in Section II [i.e., Eqn. (4) is used for the determination of MoM matrix entries when $i \neq j$ and Eqn. (6) is used when $i=j$ ], and with results obtained with the commercial software CST. Excellent agreement is observed in the comparisons carried out.

In Fig. 8(a) and (b), we show a convergence study of the results presented in Fig. 7(a) and (b) with respect to the number of BFs of (17)-(19) used to model the current density on the dipoles. These results show that four BFs (three BFs of the type shown in (17) and the BF of (19)) suffice to achieve convergence in the values of both the magnitude and phase of $R_{x x}$. In fact, the results of Fig. 8(a) and (b) indicate that the BFs of (18), which are odd in the variable $2\left(y-y_{c k}\right) / b_{k}$, do not add a relevant contribution to the current density approximation provided by the BFs of (17), which are even in the variable $2\left(y-y_{c k}\right) / b_{k}$. The fact that four BFs suffice to provide an accurate approximation of the current density on the dipoles justifies the choice of BFs carried out in this paper in accordance with the suggestions of [20]. Fig. 9(a) and (b) shows the profiles obtained for the two components of the current density on the three horizontal dipoles of the upper layer of Fig. 1(b) when the hybrid MoM

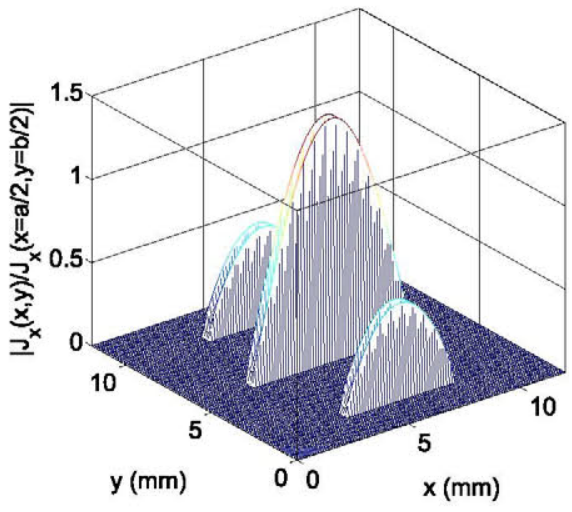

(a)

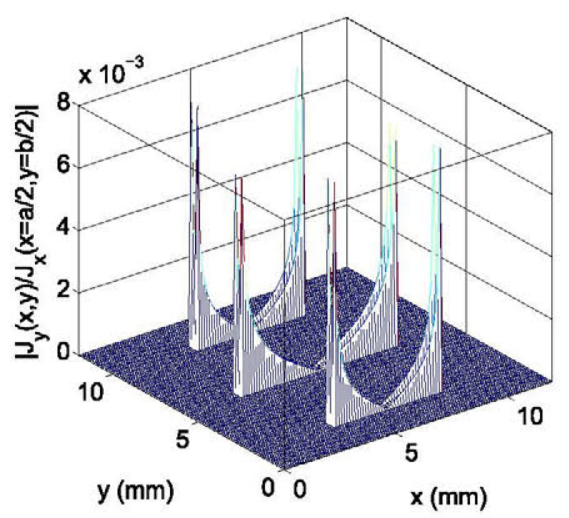

(b)

Fig. 9. (a) Normalized $x$-component and (b) $y$-component of the current density on the three horizontal dipoles of the upper layer of Fig. 1(b) Parameters: $a=b=12 \mathrm{~mm}, \theta_{\mathrm{inc}}=16.9^{\circ}, \varphi_{\mathrm{inc}}=0^{\circ}, f=11.95 \mathrm{GHz}$, dipole width $w=0.5 \mathrm{~mm}, s_{1}=s_{2}=3.5 \mathrm{~mm}, b_{11}=4.41 \mathrm{~mm}, b_{32}=7.22 \mathrm{~mm}$, $b_{22}=7.07 \mathrm{~mm}, b_{12}=4.79 \mathrm{~mm}$, and $b_{31}=b_{21}=7.6 \mathrm{~mm}$.

is applied. The profiles correspond to the periodic structure of Fig. 8(a) and (b) for which $l=7.6 \mathrm{~mm}$ at $f=11.95 \mathrm{GHz}$. Please note that the component of the current density along the dipoles direction ( $x$-component) vanishes at the dipole ends, and that its derivative at those points becomes singular. Also, the component of the current density normal to the dipoles direction ( $y$-component) becomes singular at the dipole ends. These physical edge singular behaviors are correctly accounted for by the BFs of (17)-(19). Since the normalization constant used in Fig. 9(a) and (b) is the same, Fig. 9(a) and (b) indicates that the $x$-component of the current density is on average two orders of magnitude larger than the $y$-component, and therefore, that the $y$-component is much less relevant to the $x$-component. This helps to justify why a single BF was used in the approximation of the $y$-component of the current density in (19), whereas several BFs were used in the approximation of the $x$-component in (17) and (18).

Table I shows the values of the phases of $R_{x x}$ and $R_{y y}$ in some of the structures analyzed in Fig. 7(a) and (b). These values of $\angle R_{x x}$ and $\angle R_{y y}$ are reference values, which have been obtained by means of the hybrid MoM with a sufficiently large number of BFs $\left(N_{b}=11\right)$, with a sufficiently large number of $N_{c}$ in (14) $\left(N_{c}=10\right)$, and with a sufficiently 
TABLE I

CPU TIME RATIO FOR THE COMPUTATION OF $L R_{x x}$ AND $L R_{y y}$ IN SOME of THE STRUCtURES ANALYZED IN Fig. 7. $\mathrm{T}_{\mathrm{CPU}}^{\mathrm{spe}} / \mathrm{T}_{\mathrm{CPU}}^{\text {hyb }}$ IS THE Ratio BETWEEN THE CPU TIME EMPLOYED BY THE STANDARD SPECTRAL MOM AND THAT EMPLOYED BY THE HYBRID MOM. RESULTS ARE PRESENTED FOR AN ERROR OF $5^{\circ}, 3^{\circ}$, AND $1^{\circ}$ IN THE VALUES OF $\angle R_{x x}$ AND $\angle R_{y y}$

\begin{tabular}{c|c|c|c|c|c|c}
$f$ & $l$ & $\angle R_{x x}$ & $\angle R_{y y}$ & $\begin{array}{c}\mathrm{T}_{\mathrm{CPU}}^{\mathrm{spe}} \\
\mathrm{T}_{\mathrm{CPU}}^{\mathrm{hyb}} \\
\text { [Err. } /<5^{\circ}\end{array}$ & $\begin{array}{c}\mathrm{T}_{\mathrm{CPU}}^{\mathrm{spe}} \\
\begin{array}{c}\mathrm{T}_{\mathrm{CPU}}^{\mathrm{hyb}} \\
\text { Err. } /<3^{\circ}\end{array}\end{array}$ & $\begin{array}{c}\mathrm{T}_{\mathrm{CPU}}^{\mathrm{spe}} \\
\begin{array}{c}\mathrm{T}_{\mathrm{CPU}}^{\mathrm{hyb}} \\
\text { IErr. } /<1^{\circ}\end{array}\end{array}$ \\
\hline \hline 11.95 & 7.6 & -230.7 & -225.9 & 18.6 & 69.3 & 791.7 \\
\hline 11.95 & 9.9 & -532.6 & -552.0 & 16.4 & 26.4 & 143.2 \\
\hline 11.95 & 11.3 & -703.0 & -796.0 & 38.4 & 162.8 & 8219 \\
\hline 14.0 & 6.3 & -247.3 & -238.8 & 18.8 & 151.4 & 1615 \\
\hline 14.0 & 7.4 & -416.8 & -433.4 & 18.4 & 42 & 476.8 \\
\hline 14.0 & 8.7 & -629.1 & -732.6 & 65.9 & 203.6 & 8195 \\
\hline
\end{tabular}

large number of MRW quadrature points in the numerical computation of integrals (7) and (8). Also, Table I shows the ratio between the CPU time employed by the standard spectral domain $\mathrm{MoM}$ in the computation of $\angle R_{x x}$ and $\angle R_{y y}, \mathrm{~T}_{\mathrm{CPU}}^{\mathrm{spe}}$, and that employed by the hybrid MoM of Section II, T $\mathrm{CPU}_{\text {, }}^{\text {hyb }}$, when maximum errors of $5^{\circ}, 3^{\circ}$, and $1^{\circ}$ are allowed in the computed values of $\angle R_{x x}$ and $\angle R_{y y}$ with respect to the reference values. It should be pointed out that in the computation of the hybrid MoM results, we have used interpolated MPGFs, but the time required for the interpolation of these MPGFs has not been included in $\mathrm{T}_{\mathrm{CPU}}^{\text {hyb }}$ (i.e., the coefficients $w_{\text {tupq }}^{i}$ of (16) and $h_{r s m n}^{i}$ of (15) have been precomputed and used in the determination of $G_{i}^{\mathrm{pp}, \mathrm{reg}}\left(k_{x 0}, k_{y 0}, x, y\right)$ by means of (14), but the CPU time required to compute $w_{\text {tupq }}^{i}$ and $h_{r s m n}^{i}$ has not been included in $\left.\mathrm{T}_{\mathrm{CPU}}^{\mathrm{hyb}}\right)$. And this is because the interpolation of the MPGFs is specifically devised for the analysis of a huge number of periodic structures (e.g., as required in reflectarray analysis), not for the analysis of a single periodic structure. Table I indicates that the CPU time ratio is typically between 15 and 20 for an error of $5^{\circ}$ in $\angle R_{x x}$ and $\angle R_{y y}$ (except for resonant structures containing long dipoles where a larger $\mathrm{CPU}$ time ratio is obtained), around 100 for an error of $3^{\circ}$, and around 1000 for an error of $1^{\circ}$. It is clear that the larger the accuracy required in the values of $\angle R_{x x}$ and $\angle R_{y y}$, the faster the hybrid MoM with regard to the spectral MoM. This is attributed to the fact that a huge number of terms has to be added in (4) to obtain the values of the summations with sufficient accuracy in the case $i=j$ when the spectral MoM is used (see [19, Fig. 5]). In fact, the determination of the values of $\angle R_{x x}$ and $\angle R_{y y}$ shown in Table I by means of the spectral MoM with an error lower than $1^{\circ}$ has typically required the summation of several million terms in (4) to compute $\Omega_{k l, p q}^{i i}$. This extraordinary computational effort is not necessary in the computation of $\Omega_{k l, p q}^{i i}$ with (6), which is primarily due to the analytical effort carried out to express the cross correlations of (11) and (12) in closed form (see Section II-C and Appendices). In order to give an idea of the computational efficiency of both the hybrid and the spectral MoM in the numerical analysis of the three-layered periodic structure of Fig. 1, we have obtained the values of $R_{x x}$ and $R_{y y}$

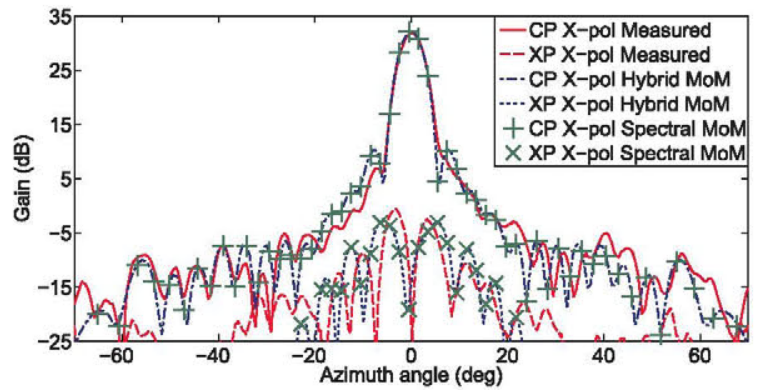

(a)

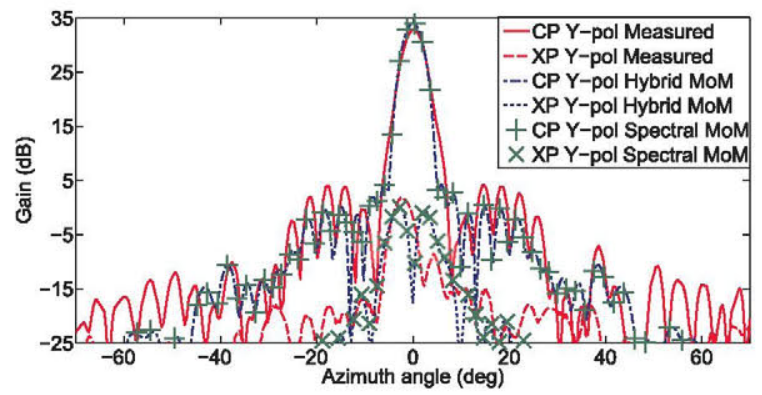

(b)

Fig. 10. Copolar and cross-polar gain patterns in the azimuth plane for the reflectarray antenna designed and measured in [15]. Results are presented at (a) $11.3 \mathrm{GHz}$ for X-polarization [15, Fig. 14.a] and (b) $14.25 \mathrm{GHz}$ for Y-polarization [15, Fig. 15.a]. The reflectarray has been analyzed with our codes, and the results obtained with both the spectral domain MoM (+ and $\times$ ) and the hybrid MoM of Section II (dotted line, and dots and dashes) under the local periodicity assumption are compared with the measurements reported in [15] (solid and dashed lines).

shown in Table I with the commercial software CST. When a maximum error of $5^{\circ}$ is allowed, we have found that CST is around 50000 times slower than the hybrid MoM, and around 3000 slower than the spectral MoM.

In Fig. 10(a) and (b), we plot results for the radiation patterns of the dual-polarization reflectarray designed in [15] for the frequency bands $11.3-12.6 \mathrm{GHz}$ (TX) and $13.75-14.25 \mathrm{GHz}(\mathrm{RX})$. This reflectarray is based on the element with two orthogonal sets of four parallel dipoles shown in Fig. 1(a) and (b). The reflectarray is circular with a 40-cm diameter, and consists of 861 elements arranged in a $33 \times 33$ square periodic grid. The dimensions of the unit cell of the grid are $a=12 \mathrm{~mm}$ and $b=12 \mathrm{~mm}$. The reflectarray is intended to generate a focused beam in the direction given by the spherical angular coordinates $\theta_{b}=16.9^{\circ}$ and $\varphi_{b}=0^{\circ}$ with respect to a system of coordinates $\left\{X_{R}, Y_{R}, Z_{R}\right\}$ with the origin at the center of the reflectarray (see [15, Fig. 1] for details). It is fed by a corrugated circular horn with phase center located at the point of coordinates $X_{R}=-193 \mathrm{~mm}, Y_{R}=0 \mathrm{~mm}$, and $Z_{R}=635 \mathrm{~mm}$. Starting from the exact dimensions obtained in [15] for the dipoles of the reflectarray elements, we have obtained the reflection matrix $\mathbf{R}$ for each of these elements under the local periodicity assumption, and from these reflection matrices, we have obtained the radiation patterns as explained in [1]. The computation of the reflection matrices has been carried out with both the standard spectral MoM and the hybrid MoM. 
TABLE II

CPU TIME RATIOS FOR THE ANALYSIS OF THE ANTENNA OF FIG. 10(a) AND (b), AND FOR THE DESIGN OF THE ANTENNAS OF FIGS. 11(a) AND (b) AND 12(a) AND (b). IN $\mathrm{T}_{\mathrm{CPU}}^{\mathrm{spe}} / \mathrm{T}_{\mathrm{CPU}}^{\mathrm{hyb}(2-\mathrm{D})}$ 2-D INTERPOLATIONS OF MPGFs ARE USED IN THE HYBRID MOM [19], AND IN $\mathrm{T}_{\mathrm{CPU}}^{\mathrm{spe}} / \mathrm{T}_{\mathrm{CPU}}^{\text {hyb(4-D) }}$, 4-D InTERPolations ARE USED (SEE SECTION II)

\begin{tabular}{c|c|c}
\hline Figure & $\mathrm{T}_{\mathrm{CPU}}^{\mathrm{spe}} / \mathrm{T}_{\mathrm{CPU}}^{\mathrm{hyb}(2 \mathrm{D})}$ & $\mathrm{T}_{\mathrm{CPU}}^{\mathrm{spe}} / \mathrm{T}_{\mathrm{CPU}}^{\mathrm{hyb}(4 \mathrm{D})}$ \\
\hline \hline Fig. 10(a) & 1.4 & 9.8 \\
\hline Fig. 10(b) & 1.6 & 11.1 \\
\hline Figs. 11(a) and (b) & 8.5 & 14.4 \\
\hline Figs. 12(a) and (b) & 9.5 & 14.2 \\
\hline
\end{tabular}

A maximum error of $7^{\circ}$ has been allowed in the phases of $R_{x x}$ and $R_{y y}$, which suffices for a graphical representation such as that shown in Fig. 10(a) and (b). Fig. 10(a) and (b) shows that the radiation patterns obtained with the spectral MoM match those obtained with the hybrid MoM, and that good agreement is found between these two sets of numerical results and measurements, especially in the neighborhood of the main beam. Table II shows two different ratios between the CPU time required for the analysis of the antenna by the standard spectral MoM and that required by the hybrid MoM. In the case of the $\mathrm{CPU}$ time ratio $\mathrm{T}_{\mathrm{CPU}}^{\mathrm{spe}} / \mathrm{T}_{\mathrm{CPU}}^{\text {hyb(2-D) }}, \mathrm{T}_{\mathrm{CPU}}^{\mathrm{spe}}$ stands for the CPU time required by the spectral MoM and $\mathrm{T}_{\mathrm{CPU}}^{\mathrm{hyb}(2-\mathrm{D})}$ stands for the CPU time required by the hybrid MoM when 2-D interpolations of the MPGFs are carried out with respect to the two spatial variables $x$ and $y$ as suggested in [19] (i.e., no interpolation is carried out with respect to $k_{x 0}$ and $k_{y 0}$, or equivalently, with respect to the incidence angles $\theta_{\text {inc }}$ and $\left.\varphi_{\text {inc }}\right)$. In the case of the CPU time ratio $\mathrm{T}_{\mathrm{CPU}}^{\mathrm{spe}} / \mathrm{T}_{\mathrm{CPU}}^{\text {hyb(4-D) }}$, $\mathrm{T}_{\mathrm{CPU}}^{\text {hyb(4-D) }}$ stands for the CPU time required by the hybrid MoM when 4-D interpolations of the MPGFs are carried out with respect to the four variables $k_{x 0}, k_{y 0}, x$, and $y$ as shown in (14). Both $\mathrm{T}_{\mathrm{CPU}}^{\text {hyb(2-D) }}$ and $\mathrm{T}_{\mathrm{CPU}}^{\text {hyb(4-D) }}$ include the CPU time required for the interpolation of the MPGFs (i.e., the CPU time required to compute the coefficients $w_{\text {tupq }}^{i}$ of (16) and $h_{r s m n}^{i}$ of (15) before (14) is ready to be used), which is around one third of the total computation time in the case of $\mathrm{T}_{\mathrm{CPU}}^{\mathrm{hyb}(4-\mathrm{D})}$. When 2-D interpolations of the MPGFs are carried out, the hybrid MoM turns out to be only $50 \%$ faster than the spectral MoM in the analysis of the antenna. However, in the case where 4-D interpolations are carried out, the hybrid MoM is around ten times faster than the spectral MoM. This important difference between the two hybrid MoM approaches has to do with the total number of MPGFs that have to be computed in each case. When one analyzes a reflectarray antenna, one different multilayered periodic problem is solved for each reflectarray element, since the dipoles dimensions and the direction of the impinging wave change from element to element. If we are using 2-D interpolations for the MPGFs, a different set of MPGFs has to be calculated to obtain the 2-D interpolated MPGF in each reflectarray element. In the particular case of the antenna analyzed in Fig. 10(a) and (b), this leads to the computation of 861 interpolated MPGFs, one per element and periodic problem. However, when we use 4-D interpolation,

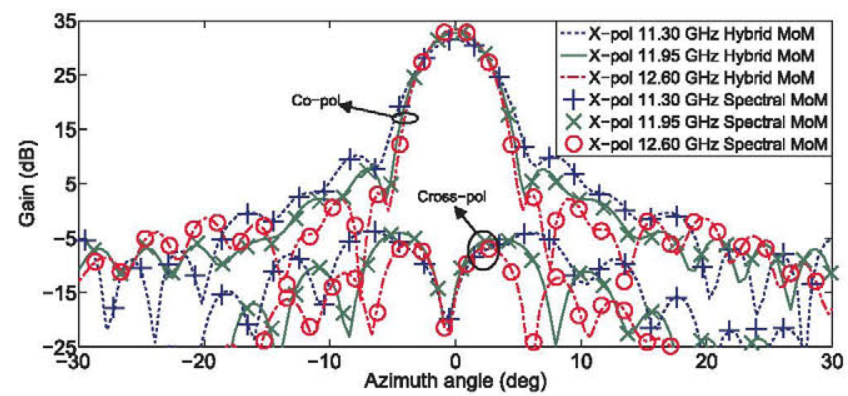

(a)

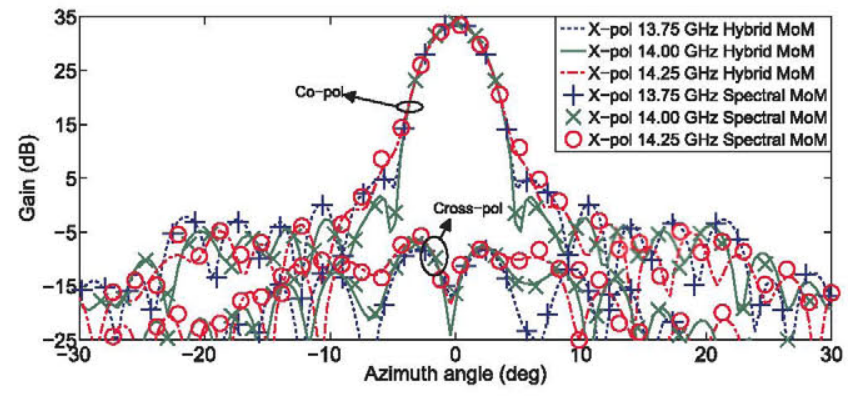

(b)

Fig. 11. Copolar and cross-polar gain patterns in the azimuth plane for the focused beam dual-polarization reflectarray antenna designed in this paper in the two bands. (a) 11.3-12.6 GHz. (b) 13.75-14.25 GHz. Results are presented in both bands for X polarization [see Figs. 10(a) and (b) of [15] for comparison]. Our results obtained with the hybrid MoM of Section II (solid and dotted lines, and dots and dashes) under the local periodicity assumption are compared with those obtained with the spectral domain MoM $\left(+, \times\right.$, and $\left.{ }^{\circ}\right)$.

we are also interpolating in terms of the angles of incidence, and the total number of MPGFs that have to be computed is reduced by more than one order of magnitude, which makes it possible important CPU time savings as shown in Table II.

The results plotted in Fig. 11(a) and (b) show the radiation patterns obtained for a dual-polarization reflectarray antenna designed from scratch with exactly the same specifications as the antenna analyzed in Fig. 10(a) and (b): the same frequency bands of operation, the same dual-polarization, the same substrate, the same grid and dimensions of the unit cell, the same feed and position of the feed, and the same focused beam pattern with the same direction of the main beam. In the design procedure, we have optimized the dimensions of the dipoles in each element to simultaneously match the required phases at central and extreme frequencies of the two frequency bands of operation $(11.3-12.6 \mathrm{GHz}$ and $13.75-14.25 \mathrm{GHz})$ by following the procedure indicated in [3], [5], and [6]. Since the starting point used in the optimization process for the antenna of Fig. 11(a) and (b) is slightly different from that used in the antenna of Fig. 10(a) and (b), the dimensions of the dipoles of the former antenna are different from those of the latter antenna, and therefore, the radiation patterns of both antennas are slightly different. The antenna of Fig. 11(a) and (b) has been designed with both the spectral MoM and the hybrid MoM, and Fig. 11(a) and (b) shows the results obtained for the radiation patterns with both approaches overlap. According to Table II, the hybrid MoM is 8.5 times faster than the spectral 
MoM in the design of the antenna when 2-D interpolations of the MPGFs are carried out, and 14.4 times faster in case 4-D interpolations of the MPGFs are carried out. This means that the hybrid MoM with 4-D interpolations of the MPGFs performs better than the hybrid MoM with 2-D interpolations of the MPGFs for the design of a reflectarray antenna (Fig. 11), but the difference of efficiency between the two approaches is not as relevant as that observed for the analysis of a reflectarray antenna (Fig. 10). The explanation for this different behavior has to do with the benefits introduced by the interpolations in each case. As mentioned previously, in the analysis of a reflectarray antenna with the hybrid MoM version of [19], the number of 2-D interpolated MPGFs that are required is equal to the number of multilayered periodic problems to be solved, i.e., one per element. In the optimized design of a reflectarray antenna, many different multilayered periodic problems with different dimensions have to be solved for each element, since the dimensions of the element have to be adjusted until the desired frequency response is achieved for that particular element. While the dimensions of the element are being adjusted (dipoles dimensions in the case of Fig. 11) and different periodic problems are being solved, the same interpolated MPGF is used all the time, which clearly favors the use of 2-D interpolated MPGFs in reflectarray antenna design with respect to reflectarray antenna analysis [19]. In case 4-D interpolations are used, the benefit is even better, since an important reduction is attained in the total number of MPGFs that have to be computed. However, the gain achieved by 4-D interpolations with regard to 2-D interpolations in reflectarray antenna design is not as relevant as that achieved in reflectarray antenna analysis (roughly a factor of two in the former case versus a factor of seven in the latter case).

In Fig. 12(a) and (b) and also in the last row of Table II, we compare the performance of the spectral MoM and the hybrid MoM in the design of a contoured beam dual-polarization reflectarray antenna based on the element with two orthogonal sets of dipoles of Fig. 1(a) and (b). The coverage requirements follow those used in [6] and [15], and the antenna is designed at the single frequency of $11.95 \mathrm{GHz}$. The reflectarray is elliptic of dimensions $111 \mathrm{~cm} \times 109 \mathrm{~cm}$, and consists of 7772 elements arranged in a $110 \times 90$ rectangular periodic grid. The dimensions of the unit cell of the grid are $a=10 \mathrm{~mm}$ and $b=12 \mathrm{~mm}$. The reflectarray is fed by a corrugated circular horn with phase center located at the point of coordinates $X_{R}=-366 \mathrm{~mm}, Y_{R}=0 \mathrm{~mm}$, and $Z_{R}=1451 \mathrm{~mm}$. Fig. 12(a) and (b) shows that the results obtained with the spectral MoM match those obtained with the hybrid MoM, and that the gain radiation patterns obtained fulfill to a large extent the templates requirements. Table II shows that the hybrid MoM is 9.5 times faster than the spectral MoM in the design of the contoured beam antenna when 2-D interpolations of the MPGFs are carried out, and 14.2 times faster in case 4-D interpolations of the MPGFs are carried out. These CPU time ratios are very similar to those obtained in the design of the focused beam antenna of Fig. 11(a) and (b). Despite the hybrid MoM approach employed (with 2-D or 4-D interpolations), Table II shows the hybrid MoM clearly outperforms the standard spectral $\mathrm{MoM}$ in the design of

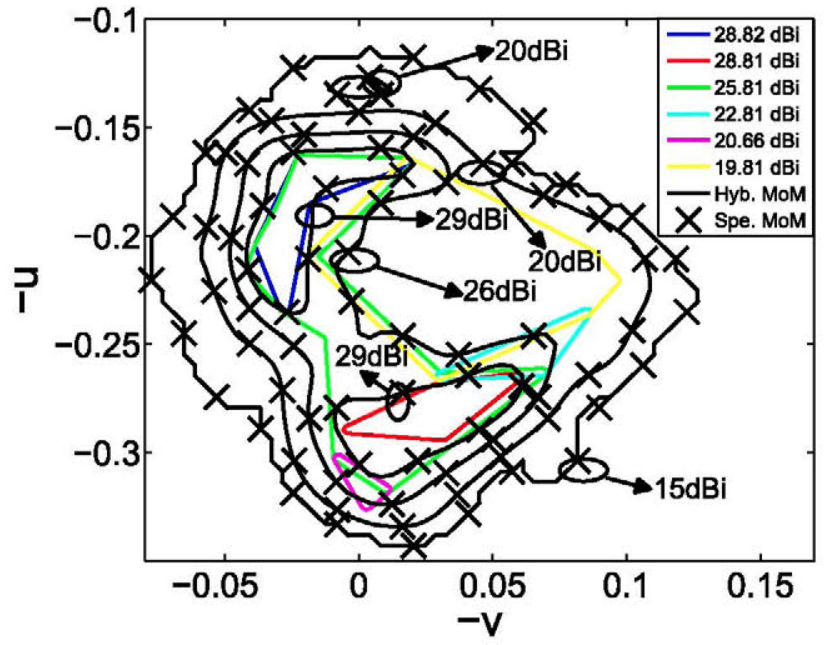

(a)

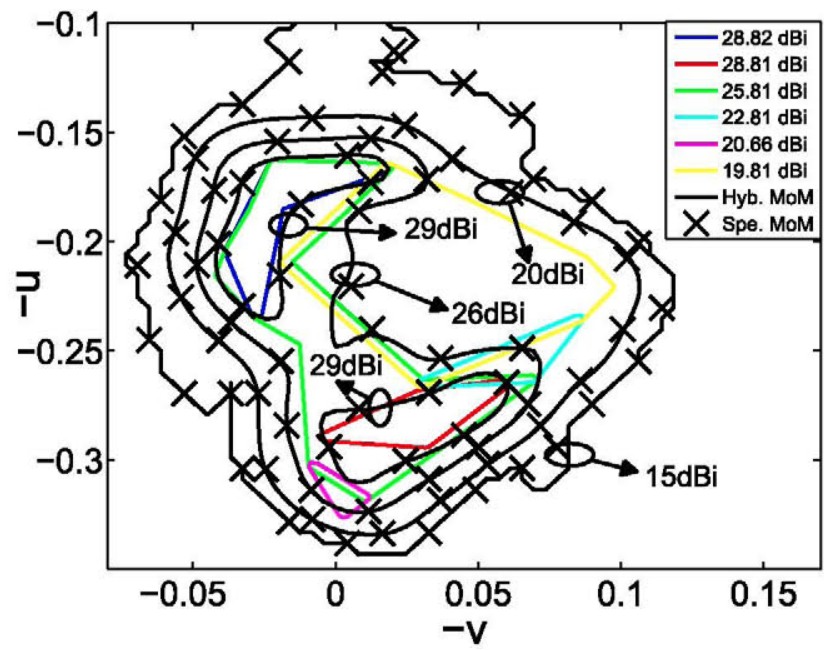

(b)

Fig. 12. Contoured lines for the copolar gain patterns of the contoured beam dual-polarization reflectarray antenna designed in this paper at $11.95 \mathrm{GHz}$. Results are presented for (a) H-polarization and (b) V-polarization. Our results obtained with the hybrid MoM of Section II (solid lines) under the local periodicity assumption are compared with those obtained with the spectral domain $\mathrm{MoM}(\times)$. The templates for South American coverage are borrowed from [6] and [15].

dual-polarization reflectarray antennas made of dipoles under the local periodicity assumption.

\section{Conclusion}

In this paper, we apply the hybrid spatial/spectral MoM approach of [19] to the analysis of multilayered periodic structures containing two orthogonal sets of parallel dipoles in the unit cell. This hybrid MoM is used in the analysis and design of dual-polarization reflectarray antennas based on the element with two orthogonal sets of dipoles. Regarding the hybrid MoM proposed in [19], two main novelties are introduced in this paper. First, the MPGFs are interpolated with great accuracy in 4-D in terms of both the spatial coordinates and the angles of incidence of the impinging wave after extraction of their behavior around the sources, and the extraction of 
spectral terms that lead to MPGFs quasi-singularities. This 4-D interpolation makes it possible an important reduction in the total number of MPGFs that has to be computed in the analysis or the design of a reflectarray antenna in comparison with the number of MPGFs required when the 2-D interpolation of [19] is used. Second, cross correlations between BFs with edge singularities are obtained in closed form in terms of complete and normal elliptic integrals of second kind for the case where the two BFs involved belong to two different coplanar dipoles. The novel hybrid MoM has been shown to be between one and three orders of magnitude faster than the standard spectral domain MoM for the analysis of multilayered periodic structures containing orthogonal sets of dipoles in the unit cell, this CPU time gain being dependent on the required accuracy. Also, the results obtained indicate the novel hybrid MoM turns out to be roughly ten times faster than the spectral MoM when applied to the reflectarray antenna analysis, and roughly fifteen times faster when applied to the reflectarray antenna design. The rationale presented in this paper for the accurate 4-D interpolation of MPGFs could still be applied for the analysis of reflectarray elements with more complex metallization patterns-crosses, loops, and so on-in periodic environments. However, in this latter case, subsectional BFs (rooftop BFs, Rao-Wilton-Glisson BFs, higher order hierarchical Legendre BFs, and so on) would be required for the approximation of the current density on the metallizations, and the cross correlations between these subsectional BFs would have to be obtained in closed form to keep the efficiency of the spatial MoM. Some promising preliminary results have already been obtained for these cross correlations between subsectional $\mathrm{BFs}$, and they will be presented in a future work.

\section{APPENDIX A}

For the definition of the parameters $a, b, c$, and $d$ that are required in the determination of $I_{p q}(z, u, v)(u \neq v, u>0$, $v>0$, and $|z|<u+v$ ), we distinguish among the following six cases.

1) For $u<v$ and $v-u \leq z<u+v$

$$
a=\frac{z+u}{v} \geq b=1>c=\frac{z-u}{v}>d=-1 .
$$

2) For $u<v$ and $u-v \leq z \leq v-u$

$$
a=1 \geq b=\frac{z+u}{v}>c=\frac{z-u}{v} \geq d=-1 .
$$

3) For $u<v$ and $-(u+v)<z \leq u-v$

$$
a=1>b=\frac{z+u}{v}>c=-1 \geq d=\frac{z-u}{v} .
$$

4) For $u>v$ and $u-v \leq z<u+v$

$$
a=\frac{z+u}{v}>b=1>c=\frac{z-u}{v} \geq d=-1 .
$$

5) For $u>v$ and $v-u \leq z \leq u-v$

$$
a=\frac{z+u}{v} \geq b=1>c=-1 \geq d=\frac{z-u}{v} .
$$

6) For $u>v$ and $-(u+v)<z \leq v-u$

$$
a=1 \geq b=\frac{z+u}{v}>c=-1>d=\frac{z-u}{v} .
$$

\section{APPENDIX B}

According to [21, eqs. (255.17) and (340.04)], the integrals of (25) can be obtained by means of the expression

$$
\begin{aligned}
J_{n}(a, b, c, d)= & \frac{2}{\sqrt{(a-c)(b-d)}} \\
& \times \sum_{j=0}^{n} \frac{a^{n-j}(-1)^{j} n !}{j !(n-j) !} C_{j}(a, b, c, d) \quad(n \geq 0)
\end{aligned}
$$

where the quantities $C_{j}(a, b, c, d)$ of (37) can be obtained by means of the recurrent expression

$$
\begin{aligned}
& C_{j}(a, b, c, d) \\
& \begin{aligned}
=\frac{1}{2(j-1)}[ & (2 j-5)(a-b)(a-c)(a-d) \\
& \times C_{j-3}(a, b, c, d)+(2 j-4)\left(2 a b-3 a^{2}\right. \\
& +2 a c-b d-b c+2 a d-c d) C_{j-2}(a, b, c, d) \\
& \left.+(2 j-3)(3 a-b-c-d) C_{j-1}(a, b, c, d)\right] \\
& (j \geq 3) .
\end{aligned}
\end{aligned}
$$

The initialization for the recurrent expression of (38) is given by

$$
\begin{aligned}
C_{0}(a, b, c, d)= & \boldsymbol{K}(k) \\
C_{1}(a, b, c, d)= & (a-b) \boldsymbol{\Pi}\left(\alpha^{2}, k\right) \\
C_{2}(a, b, c, d)= & -\frac{(a-c)(b-d)}{2} \boldsymbol{E}(k) \\
& -\frac{(a-b)(a-c)}{2} \boldsymbol{K}(k) \\
& +\frac{1}{2}(3 a-b-c-d)(a-b) \boldsymbol{\Pi}\left(\alpha^{2}, k\right)
\end{aligned}
$$

where

$$
\begin{aligned}
k & =\sqrt{\frac{(b-c)(a-d)}{(a-c)(b-d)}} \\
\alpha^{2} & =\frac{b-c}{a-c} .
\end{aligned}
$$

In (39)-(41), $\boldsymbol{K}(k)=F(\pi / 2, k)$ and $\boldsymbol{E}(k)=E(\pi / 2, k)$ stand for the complete elliptic integrals of first kind and second kind defined in [21, eqs. (110.06) and (110.07)], and $\Pi\left(\alpha^{2}, k\right)=\Pi\left(\pi / 2, \alpha^{2}, k\right)$ stands for the complete elliptic integral of the third kind defined in [21, eq. (110.08)]. Taking into account the definition of $a, b, c$, and $d$ provided in Appendix A, it turns out that $0<\alpha^{2}<k^{2}$ (hyperbolic case in the definition of the elliptic integrals of third kind according to [21, page 223]), and for this particular relation between the parameter $\alpha^{2}$ and the modulus $k, \Pi\left(\alpha^{2}, k\right)$ can be written as [21, eq. (414.01)]

$$
\begin{aligned}
\boldsymbol{\Pi}\left(\alpha^{2}, k\right)= & \boldsymbol{K}(k)+\frac{\sqrt{(a-c)(b-d)}}{a-b} \boldsymbol{K}(k) Z(\beta, k) \\
= & \boldsymbol{K}(k)+\frac{\sqrt{(a-c)(b-d)}}{a-b} \\
& \times\{\boldsymbol{K}(k) E(\beta, k)-\boldsymbol{E}(k) F(\beta, k)\}
\end{aligned}
$$


where

$$
\beta=\sin ^{-1}\left(\sqrt{\frac{b-d}{a-d}}\right)
$$

and where $Z(\beta, k)$ is the jacobian zeta function defined in [21, eq. (140.01)], and $F(\beta, k)$ and $E(\beta, k)$ are the normal elliptic integrals of first and second kind defined in [21, eqs. (110.02) and (110.03)].

\section{APPENDIX C}

Recurrence relations for $I_{p q}(z, u, v)$ can be obtained if integration by parts is applied in (21) and the recurrence relations of Bessel functions are subsequently used [32, eq. (9.127)].

In particular, once the integrals $I_{p p}(z, u, v)(p=1, \ldots, N)$ and $I_{p, p+1}(z, u, v)(p=1, \ldots, N-1)$ have all been obtained by means of the expressions (24), (25), and (37)-(45), the integrals $I_{p, p-1}(z, u, v)(p=1, \ldots, N-1)$ can be obtained by means of the recurrence relations

$$
\begin{aligned}
I_{21} & =\frac{1}{u}\left[-z I_{11}+v I_{12}\right] \\
I_{p+1, p} & =-\frac{2 z}{u} I_{p p}+\frac{v}{u}\left[I_{p, p-1}+I_{p, p+1}\right]-I_{p-1, p} \quad(p \geq 2) .
\end{aligned}
$$

Also, in order to obtain the integrals $I_{p, p+q}(z, u, v)$ for which $q \geq 2(p=1, \ldots, N-q)$, one can resort to the recurrence relations

$$
\begin{aligned}
I_{1, r+1} & =\frac{2 z}{v} I_{1 r}+\frac{2 u}{v} I_{2 r}-I_{1, r-1} \quad(r \geq 2) \\
I_{p, r+1} & =\frac{2 z}{v} I_{p r}+\frac{u}{v}\left[I_{p-1, r}+I_{p+1, r}\right]-I_{p, r-1} \quad(r>p \geq 2) .
\end{aligned}
$$

Finally, for the determination of $I_{p, p-q}(z, u, v)$ when $q \geq 2$ $(p=q+1, \ldots, N)$, one can use the recurrence relations

$$
\begin{array}{r}
I_{p+1,1}=-\frac{2 z}{u} I_{p 1}+\frac{2 v}{u} I_{p 2}-I_{p-1,1} \quad(p \geq 2) \\
I_{p+1, r}=-\frac{2 z}{u} I_{p r}+\frac{v}{u}\left[I_{p, r-1}+I_{p, r+1}\right]-I_{p-1, r} \\
\quad(p>r \geq 2) .
\end{array}
$$

\section{ACKNOWLEDGMENTS}

The authors would like to thank Prof. Fukushima from the National Astronomical Observatory of Japan for providing FORTRAN routines that efficiently implement his algorithms for the computation of complete and normal elliptic integrals of first kind and second kind.

\section{REFERENCES}

[1] J. Huang and J. A. Encinar, Reflectarray Antennas. Piscataway, NJ, USA: IEEE Press, 2008

[2] J. A. Encinar, "Design of two-layer printed reflectarrays using patches of variable size," IEEE Trans. Antennas Propag., vol. 49, no. 10, pp. $1403-1410$, Oct. 2001.

[3] J. A. Encinar and J. A. Zornoza, "Broadband design of three-layer printed reflectarrays," IEEE Trans. Antennas Propag., vol. 51, no. 7, pp. 1662-1664, Jul. 2003.
[4] J. A. Encinar and J. A. Zornoza, "Three-layer printed reflectarrays for contoured beam space applications," IEEE Trans. Antennas Propag., vol. 52, no. 5, pp. 1138-1148, May 2004.

[5] J. A. Encinar et al., "Dual-polarization dual-coverage reflectarray for space applications," IEEE Trans. Antennas Propag., vol. 54, no. 10, pp. 2828-2837, Oct. 2006.

[6] J. A. Encinar, M. Arrebola, L. F. de La Fuente, and G. Tosso, "A transmit-receive reflectarray antenna for direct broadcast satellite applications," IEEE Trans. Antennas Propag., vol. 59, no. 9, pp. 3255-3264, Sep. 2011.

[7] S. Dieter, C. Fischer, and W. Menzel, "Single-layer unit cells with optimized phase angle behavior," in Proc. 3rd Eur. Conf. Antennas Propag. EuCAP, Mar. 2009, pp. 1149-1153.

[8] L. Li et al., "Novel broadband planar reflectarray with parasitic dipoles for wireless communication applications," IEEE Antennas Wireless Propag. Lett., vol. 8, pp. 881-885, Jul. 2009.

[9] K. H. Sayidmarie and M. E. Bialkowski, "Fractal unit cells of increased phasing range and low slopes for single-layer microstrip reflectarrays," IET Microw. Antennas Propag., vol. 5, no. 11, pp. 1371-1379, 2011.

[10] R. Florencio, R. R. Boix, V. Losada, J. A. Encinar, E. Carrasco, and M. Arrebola, "Comparative study of reflectarrays based on cells with three coplanar dipoles and reflectarrays based on cells with three stacked patches," in Proc. 6th Eur. Conf. Antennas Propag. EuCAP, Prague, Czech Republic, Apr. 2012, pp. 3707-3710.

[11] E. Carrasco, M. Barba, J. A. Encinar, M. Arrebola, F. Rossi, and A. Freni, "Design, manufacture and test of a low-cost shaped-beam reflectarray using a single layer of varying-sized printed dipoles," IEEE Trans. Antennas Propag., vol. 61, no. 6, pp. 3077-3085, Jun. 2013.

[12] R. Florencio, R. R. Boix, E. Carrasco, J. A. Encinar, M. Barba, and G. Pérez-Palomino, "Broadband reflectarrays made of cells with three parallel dipoles," Microw. Opt. Technol. Lett., vol. 56, no. 3, pp. 748-753, Mar. 2014.

[13] K. C. Clancy, M. E. Cooley, and D. Bressler, "Apparatus and method for reducing polarization cross-coupling in cross-dipole reflectarrays," U.S. Patent, U.S. Patent 20010050653 A1, Dec. 13, 2001.

[14] R. Florencio, J. A. Encinar, R. R. Boix, and G. Pérez Palomino, "Dual-polarization reflectarray made of cells with two orthogonal sets of parallel dipoles for bandwidth and cross-polarization improvement," IET Microw. Antennas Propag., vol. 8, no. 15, pp. 1389-1397, 2014.

[15] R. Florencio, J. A. Encinar, R. R. Boix, V. Losada, and G. Toso, "Reflectarray antennas for dual polarization and broadband telecom satellite applications," IEEE Trans. Antennas Propag., vol. 63, no. 4, pp. 1234-1246, Apr. 2015.

[16] D. M. Pozar and T. A. Metzler, "Analysis of a reflectarray antenna using microstrip patches of variable size," Electron. Lett., vol. 29, no. 8, pp. 657-658, Apr. 1993.

[17] D. M. Pozar, S. D. Targonski, and H. D. Syrigos, "Design of millimeter wave microstrip reflectarrays," IEEE Trans. Antennas Propag., vol. 45, no. 2, pp. 287-296, Feb. 1997.

[18] M. Zhou, S. B. Sørensen, O. S. Kim, E. Jørgensen, P. Meincke, and O. Breinbjerg, "Direct optimization of printed reflectarrays for contoured beam satellite antenna applications," IEEE Trans. Antennas Propag., vol. 61, no. 4, pp. 1995-2004, Apr. 2013.

[19] R. Florencio, R. R. Boix, and J. A. Encinar, "Fast and accurate MoM analysis of periodic arrays of multilayered stacked rectangular patches with application to the design of reflectarray antennas," IEEE Trans. Antennas Propag., vol. 63, no. 6, pp. 2558-2571, Jun. 2015.

[20] A. M. Lerer and A. G. Schuchinsky, "Full-wave analysis of threedimensional planar structures," IEEE Trans. Microw. Theory Techn., vol. 41, no. 11, pp. 2002-2015, Nov. 1993.

[21] P. F. Byrd and M. D. Friedman, Handbook of Elliptic Integrals for Engineers and Scientists, 2nd ed. New York, NY, USA: Springer-Verlag, 1971.

[22] T. Fukushima, "Precise and fast computation of elliptic integrals and functions," in Proc. IEEE 22nd Symp. Comput. Arithmetic, Jun. 2015, pp. 50-57, doi :10.1109/ARITH.2015.15.

[23] E. Martinez-de-Rioja, J. A. Encinar, R. Florencio, and R. R. Boix, "Dual polarized reflectarray antenna to generate independent beams in $\mathrm{Ku}$ and Ka bands," in Proc. 10th Eur. Conf. Antennas Propag. (EuCAP), Apr. 2016, pp. 1-5, doi: 10.1109/EuCAP.2016.7481439

[24] R. Florencio, R. R. Boix, and J. A. Encinar, "Enhanced MoM analysis of the scattering by periodic strip gratings in multilayered substrates," IEEE Trans. Antennas Propag., vol. 61, no. 10, pp. 5088-5099, Oct. 2013. 
[25] F. Mesa, R. Marques, and M. Horno, "A general algorithm for computing the bidimensional spectral Green's dyads in multilayered complex bianisotropic media: The equivalent boundary method," IEEE Trans. Microw. Theory Tech., vol. 39, no. 9, pp. 1640-1649, Sep. 1991.

[26] J. R. Mosig, "Integral equation technique," in Numerical Techniques for Microwave and Millimeter-Wave Passive Structures, T. Itoh, Ed. New York, NY, USA: Wiley, 1989, pp. 133-213.

[27] D. M. Pozar and D. H. Schaubert, "Scan blindness in infinite phased arrays of printed dipoles," IEEE Trans. Antennas Propag., vol. AP-32, no. 6, pp. 602-610, Jun. 1984.

[28] L. S. Gradshteyn and L. M. Ryzhik, Table of Integrals, Series and Products, 6th ed. San Diego, CA, USA: Academic, 2000.

[29] W. H. Press, S. A. Teukolsky, W. T. Vetterling, and B. P. Flannery, Numerical Recipes in FORTRAN-The Art of Scientific Computing, 2nd ed. Cambridge, U.K.: Cambridge Univ. Press, 1992.

[30] J. Ma, V. Rokhlin, and S. Wandzura, "Generalized Gaussian quadrature rules for systems of arbitrary functions," SIAM J. Numer. Anal., vol. 33, no. 3, pp. 971-996, Jun. 1996.

[31] B. A. Munk, Frequency Selective Surfaces. New York, NY, USA: Wiley, 2000.

[32] M. Abramowitz and I. Stegun, Handbook of Mathematical Functions, 9th ed. New York, NY, USA: Dover, 1970. 